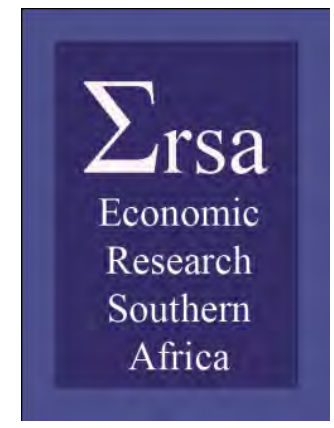

\title{
A decision-theoretic model of asset-price underreaction and overreaction to dividend news
}

\author{
Alexander Ludwig and Alexander Zimper \\ ERSA working paper 296 \\ http://econrsa.org/home/index.php?option=com_docman\&task=doc_download\&gid=440\&Itemid=67
}

June 2012

Economic Research Southern Africa (ERSA) is a research programme funded by the National Treasury of South Africa.

The views expressed are those of the author(s) and do not necessarily represent those of the funder, ERSA or the author's affiliated institution(s). ERSA shall not be liable to any person for inaccurate information or opinions contained herein. 


\title{
A decision-theoretic model of asset-price underreaction and overreaction to dividend news
}

\author{
Alexander Ludwig* Alexander Zimper ${ }^{\dagger}$
}

June 13, 2012

\begin{abstract}
We combine new developments in decision theory with a standard consumptionbased asset-pricing framework. In our model the efficient market hypothesis is violated if and only if agents' beliefs express ambiguity about the stochastic process driving economic fundamentals. Asset price fluctuations result because agents with ambiguous beliefs are prone to a confirmatory bias in the interpretation of new information. We demonstrate that our approach gives rise to price-patterns of "underreaction" and "overreaction" to news about dividend payments. Although these empirical phenomena have received significant attention in the behavioral finance literature, we argue that our decision-theoretic underpinning of psychological attitudes has a less ad hoc flavor than existing approaches.
\end{abstract}

Keywords: Choquet Expected Utility Theory, Portfolio Choice, Asset Pricing Puzzles

JEL Classification Numbers: C62, D81, G11, G12

${ }^{*}$ CMR, University of Cologne; Albertus-Magnus-Platz; 50923 Köln; Germany; Email: ludwig@wiso.unikoeln.de.

${ }^{\dagger}$ Department of Economics, University of Pretoria, Private Bag X20, Hatfield 0028, South Africa. Email: alexander.zimper@up.ac.za 


\section{Introduction}

\subsection{Motivation}

As a consequence of the recent financial markets crisis and the bubbles in housing and stock markets that preceded it, there exists renewed interest in understanding better the forces that are behind such drastic movements of asset prices. Several empirical price patterns suggest that asset price dynamics cannot be satisfactorily explained by rational expectations models alone. According to such models asset prices are derived from the portfolio optimization problems of agents who fully understand stochastic processes that drive economic fundamentals. The common theme of rational expectation models is the efficient market hypothesis $(=\mathrm{EMH})$ which implies that price fluctuations should only reflect payoff-relevant changes in agents' information about economic fundamentals.

The present paper deviates from this common theme. We develop a decision theoretic model which gives rise to "underreaction" and "overreaction" of asset prices to dividend news. These pricing phenomena stand for persistent empirical violations of the EMH. ${ }^{1}$ Consider the process of equilibrium returns for a given asset $\left(R_{t}\right)_{t \geq 0}$ such that

$$
R_{t+1}=\frac{p_{t+1}^{*}+Y_{t+1}}{p_{t}^{*}} \text { for all } t \geq 1
$$

whereby $\left(p_{t}^{*}\right)_{t \geq 0}$ denotes the ex-dividend equilibrium price process and $\left(Y_{t}\right)_{t \geq 0}$ denotes the dividend process. Let $G_{t}$, respectively $B_{t}$, denote the event that there is "good", respectively "bad", news in period $t$. Furthermore, we denote by $\pi$ the probability measure that governs the "true" stochastic processes of economic fundamentals. We formally define underreaction and overreaction as follows.

- Underreaction to a sequence of $k+1$ good news:

$$
E\left[R_{t+1}, \pi\left(\omega \mid B_{t-k-1}, G_{t-k}, \ldots, G_{t}\right)\right]>E\left[R_{t+1}, \pi\left(\omega \mid G_{t-k-1}, B_{t-k}, \ldots, B_{t}\right)\right] .
$$

- Overreaction to a sequence of $k+1$ good news:

$$
E\left[R_{t+1}, \pi\left(\omega \mid B_{t-k-1}, G_{t-k}, \ldots, G_{t}\right)\right]<E\left[R_{t+1}, \pi\left(\omega \mid G_{t-k-1}, B_{t-k}, \ldots, B_{t}\right)\right] .
$$

\footnotetext{
${ }^{1}$ For empirical evidence on underreaction see, e.g., Bernard and Thomas (1990), Cutler et al. (1991), Bernard (1992), Jegadeesh and Titman (1993), Rouwenhorst (1997), Chan et al. (1997) and Chan (2003). For empirical evidence on overreaction compare, e.g., De Bondt and Thaler (1985), Zarowin (1989), Chopra et al. (1992), La Porta (1996), La Porta et al. (1997), and Antweiler and Frank (2006).
} 
Barberis, Shleifer and Vishny (1998) argue that the typical empirical phenomenon is underreaction to a single period, i.e., $k=0$, and overreaction to a sequence, i.e., $k \geq 1$, of good news. Following Barberis et al. (1998), we are therefore especially interested in identifying conditions such that the following return pattern emerges from our asset pricing model.

- Underreaction to a single period of good news:

$$
E\left[R_{t+1}, \pi\left(\omega \mid B_{t-1}, G_{t}\right)\right]>E\left[R_{t+1}, \pi\left(\omega \mid G_{t-1}, B_{t}\right)\right]
$$

- Overreaction to multiple periods of good news: For all $k \geq 1$,

$$
E\left[R_{t+1}, \pi\left(\omega \mid B_{t-k-1}, G_{t-k}, \ldots, G_{t}\right)\right]<E\left[R_{t+1}, \pi\left(\omega \mid G_{t-k-1}, B_{t-k}, \ldots, B_{t}\right)\right]
$$

The interpretation of the empirical phenomena (4) and (5) is straightforward. For a single period of good news, the present period's asset price does not incorporate the good news sufficiently, i.e., $p_{t}^{*}$ tends to underreact to good news that come as a surprise. Consequently, the average future return is greater for a positive than for a negative surprise. For multiple periods of good news the opposite effect happens: now the present period's asset price tends to overreact to a sequence of good news, i.e., to good news that are no longer surprising. Without, arguably, unrealistic non-stationarity assumptions about the objective dividend process, the EMH cannot explain (4) and (5). To see this consider the interesting benchmark case according to which today's news and future economic fundamentals (=dividends) are independent and identically distributed. Under this i.i.d. assumption, the EMH implies that the lhs and rhs in (2), as well as in (3), must hold with equality for any number $k$. As a consequence, rational expectation models of asset pricing - which assume that the objective probability measure $\pi$ coincides with the economic agents' subjective beliefs-are unable to generate the return patterns (4) and (5) for this i.i.d. benchmark case. In contrast, our behavioral model of asset price fluctuations will be able to generate the return patterns (4) and (5) even if $\pi$ gives rise to i.i.d. dividend payments.

We formalize the subjective beliefs of a representative economic agent within the axiomatic framework of Choquet expected utility (CEU) theory (Schmeidler 1986, 1989; Gilboa 1987). In contrast to standard expected utility (EU) theory, beliefs of a CEU agent 
are described by non-additive rather than additive probability measures. These accommodate violations of Savage's (1954) sure thing principle as demonstrated by Ellsberg (1961) paradoxes. Ellsberg paradoxes have been elicited for preferences over Savage acts. These assign to every state of the world some deterministic consequence, e.g., some infinite stream of monetary payoffs. The choice between Savage acts can be interpreted as an investment choice between different asset-portfolios. Any model of preferences under uncertainty that incorporates Ellsberg-type behavior is therefore relevant for real-life investment decisions on financial markets. ${ }^{2}$

To simplify our analysis we further restrict attention to neo-additive capacities in the sense of Chateauneuf, Eichberger and Grant (2007). Accordingly, an agent's non-additive belief about the likelihood of an event is a weighted average of an ambiguous part and an additive part, i.e., some subjective additive probability measure. In a next step we use our decision-theoretic model to formalize our central behavioral assumption. It states that agent's belief generation process is governed by a confirmatory bias heuristic: the interpretation of new information depends on the asset's past performance in terms of dividend payments. Our formal concept of a confirmatory bias heuristic exploits the fact that there exist several perceivable Bayesian update rules for non-additive probability measures. These reflect different psychological attitudes towards the interpretation of new information. ${ }^{3}$ We consider the optimistic and pessimistic (Gilboa and Schmeidler 1993; Sarin and Wakker 1998a) as well as the full Bayesian update rule (Pires 2002; Eichberger, Grant, and Kelsey 2006; Siniscalchi 2011). Depending on the agent's information about the asset's past performance in terms of dividend payments, three corresponding asset pricing regimes emerge whereby frequency of change between asset pricing regimes is governed by a switching parameter with value $n \geq 1$. Our formal approach thus captures the intuitive notion that good news result in an optimistic whereas bad news result in a pessimistic bias in the agent's belief about the asset's future prospects. Whenever neo-additive beliefs of our approach reduce to additive probability measures, these three different pricing regimes collapse into the familiar asset pricing model of expected utility theory with additively time-separable preferences. However, when neo-additive beliefs of our model express ambiguity attitudes, the existence of three different pricing regimes results in a higher fluctuation of equilibrium prices than for standard expected utility.

\footnotetext{
${ }^{2}$ Also note that $\mathrm{Wu}$ and Gonzalez (1999) report Ellsberg-type behavior (more specifically: inversely $S$-shaped decision weighting functions) for bets on the future value of the Dow Jones Industrial Average.

${ }^{3}$ As explained in Section 2, this "indeterminacy" of update rules is a direct consequence of the violation of Savage's (1954) sure thing principle as elicited in paradoxes of the Ellsberg (1961) type.
} 
As our paper's main formal contribution we characterize conditions for which our model generates asset-return patterns (2) and (3), respectively. In particular, we pin down $n=2$ as the unique value of the switching parameter that simultaneously generates the empirical phenomena (4) and (5) within our model.

\subsection{Our modeling choice: Dynamic inconsistency combined with sequential naïvety}

The decision-theoretic principles of consequentialism, dynamic consistency, and universalism give rise to standard EU theory whenever dynamic decision situations are reduced to a static Savage framework. In this framework ex ante and ex post preferences are defined over Savage acts (Sarin and Wakker 1998b; Ghirardato 2002). Loosely speaking ${ }^{4}$, consequentialism states that an agent's preferences over future consequences should not be affected by the way he arrived at a decision situation; dynamic consistency states that there should be no conflict of interest between the agent and his future selves; and universalism states that behavioral axioms have to apply to all - and not only to conveniently selected - events.

Starting with the seminal contribution of Epstein and Wang (1994) existing models of intertemporal asset pricing under ambiguity are typically formalized within the (consequentialist) rectangular multiple priors framework (Sarin and Wakker 1998b, Epstein and Schneider 2003). The rectangular multiple priors approach adds dynamic consistency as an axiom to a multi-period version of the Gilboa and Schmeidler (1989) multiple priors framework. ${ }^{5}$ To this end, it has to give up universalism in order to avoid reduction to standard EU theory. Dynamic consistency is ensured by fixing an information filtration such that any violation of Savage's sure thing principle can only happen at events that may never be observed by the decision maker. By construction, the rectangular multiple priors approach thereby comes at the cost of excluding any dynamic version of Ellsberg paradoxes. As a consequence, only a rather restrictive notion of ambiguity attitudes remains admissible (cf.

\footnotetext{
${ }^{4}$ In Section 2 we give precise definitions of these principles and derive their formal relationship to Savage's sure thing principle which is at the heart of (subjective) EU theory.

${ }^{5}$ Observe that an CEU decision maker of our model who resolves his ambiguity exclusively in a pessimistic way (i.e., via a convex neo-additive capacity) could be equivalently described within Gilboa and Schmeidler's (1989) multiple priors framework if the priors are given as the core of this neo-additive capacity. However, in contrast to the original Gilboa and Schmeidler (1989) multiple priors framework - which can only express ambiguity aversion - our approach is in line with empirical evidence that suggests a mix between ambiguity proneness and ambiguity aversion (cf. the inversely $S$-shaped weighting functions as described by Wu and Gonzales (1996, 1999) and by Wakker (2004)).
} 
Ghirardato 2002; Hansen, Sargent, Turmuhambetova, and Williams 2006; Siniscalchi 2011; and Section 2.2. of this paper).

In contrast, the (consequentialist) decision-theoretic framework of our paper satisfies universalism. The representative agent of our model expresses ambiguity attitudes if and only if his decision behavior is dynamically inconsistent. As one major difference to the rectangular multiple priors approach, our decision-theoretic model may thus express ambiguity attitudes which are independent of any fixed information partition. In particular, violations of the sure thing principle may also occur at observable events. As another major difference, our confirmatory bias heuristic is formalized in terms of three different Bayesian update rules. In contrast, the rectangular multiple priors approach is based on a unique (measure-by-measure) Bayesian update rule. Unless the rectangular multiple priors framework could be extended to a version that admits for different Bayesian updates rules, our approach of modeling under- respectively overreaction through an application of different psychologically motivated Bayesian update rules is not possible within this dynamically consistent framework.

For dynamically inconsistent agents there exist two alternative ways to characterize equilibrium conditions. One alternative is to consider agents who are sequentially rational (i.e., sophisticated) in the sense that they fully understand their dynamically inconsistent decision behavior so that an ex ante agent correctly anticipates his future selves' decisions. An according model of intertemporal asset pricing with dynamically inconsistent but sequentially rational CEU agents is described in Zimper (2012). He combines the notion of subgame-perfectness with the standard general equilibrium approach to take account of conflict of interest between an ex ante agent and his future selves.

In this paper we have chosen the alternative approach where sequentially naïve agents are not aware of their dynamically inconsistent decision behavior. Sequentially naïve agents of our model solve their intertemporal decision problem - in terms of an infinite contingent plan of actions - without realizing that their future selves might have a strict incentive to deviate from an ex ante optimal plan of actions. The resulting first order condition determining the current period's equilibrium price is thereby formally equivalent to a two period maximization problem according to which myopic agents only think one period ahead. The asset pricing equilibrium of our model - with sequentially naïve agents who care about infinite consumption streams - could therefore be interpreted as the equilibrium of an economy consisting of an infinite sequence of myopic agents. Empirical evidence favoring asset pricing models with myopic agents is provided, e.g., by Bernartzi and Thaler 
(1995), Thaler, Tversky, Kahneman, and Schwartz (1997) as well as LeBaron (2000, 2006). ${ }^{6}$ This supports our modeling choice.

The remainder of our analysis is structured as follows. Section 2 describes the decisiontheoretic foundations of our model. We emphasize conceptional differences between our approach and the rectangular multiple priors model of Epstein and Schneider (2003). Based on these decision-theoretic preliminaries, Section 3 formalizes our behavioral assumptions: agents are (i) prone to a confirmatory bias and (ii) naïve in the sense that they are ignorant with respect to this bias. Section 4 merges our assumptions on expectations and behavior with a standard Lucas (1978) type asset pricing model. Section 5 revisits the formal definitions of under- and overreaction and derives conditions under which our asset-pricing model generates these return patterns. In Section 6 we illustrate-by use of a simple numerical example - the equilibrium return patterns. In Section 7 we discuss merits and shortcomings of our approach relative to the existing literature on under- and overpricing. Finally, Section 8 concludes. The formal proof of our proposition is relegated to a separate appendix.

\section{Decision-theoretic preliminaries}

The formalism of our behavioral model is based on Choquet decision theory. In the first subsection we recall basic elements of Choquet expected utility (CEU) theory and we introduce neo-additive capacities as a specific class of non-additive probability measures. In the second subsection we discuss the relationship between dynamic consistency and Savage's sure thing principle. The analysis follows closely Zimper $(2011,2012)$. The final subsection presents different Bayesian update rules for non-additive probability measures.

\subsection{Choquet decision theory and neo-additive capacities}

CEU theory was first axiomatized by Schmeidler $(1986,1989)$ within the Anscombe and Aumann (1963) framework, which assumes preferences over objective probability distributions. Subsequently, Gilboa (1987) has presented an CEU axiomatization within the Savage (1954) framework, assuming a purely subjective notion of likelihood. When restricted to the domain of gains, CEU theory is formally equivalent to cumulative prospect theory (Tversky and Kahneman 1992; Wakker and Tversky 1993) which generalizes the celebrated prospect theory of Kahneman and Tversky (1979). Moreover, as a representation of preferences over

\footnotetext{
${ }^{6} \mathrm{We}$ are grateful to an anonymous referee who pointed us to this literature.
} 
lotteries, CEU theory coincides with rank dependent utility theory as introduced by Quiggin $(1981,1982)$. Within the context of CEU theory, properties of such capacities are used in the literature for formal definitions of ambiguity and uncertainty attitudes (Schmeidler 1989; Epstein 1999; Ghirardato and Marinacci 2002), pessimism and optimism (Eichberger and Kelsey 1999; Wakker 2001), as well as likelihood insensitivity (Wakker 2004, 2010).

Let us consider a measurable space $(\Omega, \mathcal{F})$ with $\mathcal{F}$ denoting a $\sigma$-algebra on the state space $\Omega$ and a non-additive probability measure (=capacity) $\nu: \mathcal{F} \rightarrow[0,1]$ satisfying

(i) $\nu(\emptyset)=0, \nu(\Omega)=1$

(ii) $A \subset B \Rightarrow \nu(A) \leq \nu(B)$ for all $A, B \in \mathcal{F}$.

The Choquet integral of an $\mathcal{F}$-measurable function $f: \Omega \rightarrow \mathbb{R}$ with respect to capacity $\nu$ is defined as the following Riemann integral extended to domain $\Omega$ (Schmeidler 1986):

$$
E[f, \nu(d \omega)]=\int_{-\infty}^{0}(\nu(\{\omega \in \Omega \mid f(\omega) \geq z\})-1) d z+\int_{0}^{+\infty} \nu(\{\omega \in \Omega \mid f(\omega) \geq z\}) d z
$$

whereby we simply write $E[f, \nu]$ for $E[f, \nu(d \omega)]$. For example, assume that $f$ takes on $m$ different values such that $A_{1}, \ldots, A_{m}$ is the unique partition of $\Omega$ with $f\left(\omega_{1}\right)>\ldots>f\left(\omega_{m}\right)$ for $\omega_{i} \in A_{i}$. Then the Choquet expectation (6) becomes

$$
E[f, \nu]=\sum_{i=1}^{m} f\left(\omega_{i}\right) \cdot\left[\nu\left(A_{1} \cup \ldots \cup A_{i}\right)-\nu\left(A_{1} \cup \ldots \cup A_{i-1}\right)\right] .
$$

Our approach focuses on non-additive probability measures that are defined as neoadditive capacities in the sense of Chateauneuf, Eichberger and Grant (2007).

Definition. Fix some set of null-events $\mathcal{N} \subset \mathcal{F}$ for the measurable space $(\Omega, \mathcal{F}){ }^{7}$ The neo-additive capacity, $\nu$, is defined, for some $\delta, \lambda \in[0,1]$ by

$$
\nu(A)=\delta \cdot \nu_{\lambda}(A)+(1-\delta) \cdot \pi(A)
$$

for all $A \in \mathcal{F}$ such that $\pi$ is some additive probability measure satisfying

$$
\pi(A)= \begin{cases}0 & \text { if } A \in \mathcal{N} \\ 1 & \text { if } \Omega \backslash A \in \mathcal{N}\end{cases}
$$

\footnotetext{
${ }^{7}$ Intuitively speaking, null events are the events that the decision maker deems impossible. For instance, within a Savage framework we have that $A \in \mathcal{N}$ if and only if, for all Savage acts $f, g, h, h^{\prime}$,

$$
h_{A} f \succeq h_{A}^{\prime} g \Leftrightarrow f \succeq g,
$$

i.e., consequences on $A$ are irrelevant to the decision maker; (see Subsection 2.2. for the notation).
} 
and the non-additive probability measure $\nu_{\lambda}$ is defined as follows

$$
\nu_{\lambda}(A)= \begin{cases}0 & \text { iff } A \in \mathcal{N} \\ \lambda & \text { else } \\ 1 & \text { iff } \Omega \backslash A \in \mathcal{N} .\end{cases}
$$

Throughout this paper, we restrict attention to sets of null-events $\mathcal{N}$ such that $A \in \mathcal{N}$ if and only if $\pi(A)=0$, which implies $\nu(A)=0$ (resp. $\nu(A)=1)$ if and only if $\pi(A)=0$ (resp. $\pi(A)=1$ ). For any essential events, i.e., $A \notin \mathcal{N}$ and $\Omega \backslash A \notin \mathcal{N}$, the neo-additive capacity $\nu$ in $(7)$ then simplifies to

$$
\nu(A)=\delta \cdot \lambda+(1-\delta) \cdot \pi(A)
$$

with $0<\pi(A)<1$. Neo-additive capacities can thus be interpreted as non-additive beliefs that stand in for deviations from additive beliefs such that a parameter $\delta$ (degree of ambiguity) measures the lack of confidence the decision maker has in some subjective additive probability distribution $\pi$.

The following observation extends a result (Lemma 3.1) of Chateauneuf, Eichberger and Grant (2007) for finite random variables to the more general case of random variables with a compact range (for a formal proof see Zimper 2012).

Observation 1. Let $f: \Omega \rightarrow \mathbb{R}$ be an $\mathcal{F}$-measurable function with compact range. The Choquet expected value (6) of $f$ with respect to a neo-additive capacity (7) is then given by

$$
E[f, \nu]=\delta\left(\lambda \max _{\omega \in \Omega} f(\omega)+(1-\lambda) \min _{\omega \in \Omega} f(\omega)\right)+(1-\delta) E[f, \pi] .
$$

According to Observation 1, the Choquet expected value of a random variable $f$ with respect to a neo-additive capacity is a convex combination of the expected value of $f$ with respect to some additive probability measure $\pi$ and an ambiguity part. If there is no ambiguity, i.e., $\delta=0$, then the Choquet expected value (9) reduces to the standard expected value of a random variable with respect to an additive probability measure. In case there is some ambiguity, however, the second parameter $\lambda$ measures how much weight the decision maker puts on the best possible outcome of $f$ when resolving his ambiguity. Conversely, $(1-\lambda)$ is the weight he puts on the worst possible outcome of $f$. As a consequence, we 
interpret $\lambda$ as a (relative) "optimism under ambiguity" parameter whereby $\lambda=1$, resp. $\lambda=0$, corresponds to extreme optimism, resp. extreme pessimism.

Remark. In addition to the above "motivational" interpretation in terms of ambiguity attitudes, there exists a "cognitive" interpretation of neo-additive beliefs in terms of "likelihood insensitivity" (Wakker 2004, 2010). Likelihood insensitivity is a persistent experimental phenomenon which "reflects diminishing sensitivity for a scale bounded from two sides" (Wakker 2010, p. 227). Accordingly, the decision maker does not sufficiently distinguish between probabilities that lie between zero and one (for example, any uncertainty is resolved in a fifty-fifty way).

\subsection{Sure thing principle, consequentialism, and dynamic consis- tency}

Under the standard Bayesian assumption, dynamic decision situations can equivalently be described within a static Savage framework in which ex ante and ex post preferences are defined over Savage acts. The sure thing principle, defined for ex ante preferences, then bears implications on principles of dynamic decision making (e.g., dynamic consistency) which link ex ante with ex post preferences. This subsection analyzes the relationship between the sure thing principle and dynamic consistency. We conclude this subsection with a discussion of the rectangular multiple priors approach.

Consider again the state space $\Omega$ with $\sigma$-algebra $\mathcal{F}$ which contains all events that are relevant to the decision maker in the sense that he attaches probabilities to these events. For example, we might simply follow Savage (1954) and assume that $\mathcal{F}$ is given as the power-set $2^{\Omega}$. Recall that a Savage act $f$ is an $\mathcal{F}$-measurable function that maps the state space $\Omega$ into an arbitrary set of consequences $X$, i.e., $f: \Omega \rightarrow X$. Event space $\mathcal{F}$ is supposed to be rich enough to cover all aspects of uncertainty relevant to the decision maker. Whenever $\omega \in \Omega$ is the (unique) true state of the world and the agent has chosen Savage act $f$, he ends up with consequence $f(\omega)$ after uncertainty is resolved. Given two Savage acts $f, g$, let $B, \neg B \in \mathcal{F}$ denote two complementary events and define Savage act $f_{B} g: \Omega \rightarrow X$ as follows:

$$
f_{B} g(\omega)=\left\{\begin{array}{l}
f(\omega) \quad \text { for } \omega \in B \\
g(\omega) \text { for } \omega \in \neg B .
\end{array}\right.
$$

Ex ante preferences over Savage acts, denoted $\succeq$, are interpreted as the decision maker's preferences before he receives any information. 
Fix now some partition $\Pi$ of $\Omega$ whereby $\sigma(\Pi) \subseteq \mathcal{F}$ denotes the $\sigma$-algebra generated by $\Pi$.

Definition: $\sigma(\Pi)$-sure thing principle. For all Savage acts $f, g, h, h^{\prime}$ and all events $B \in \sigma(\Pi)$, the following condition holds for ex ante preferences:

$$
f_{B} h \succeq g_{B} h \Rightarrow f_{B} h^{\prime} \succeq g_{B} h^{\prime} .
$$

Turn now to ex post preferences over Savage acts, denoted $\succeq_{B}$. These are interpreted as preferences conditional on $B$, i.e., after the decision maker has observed the occurrence of some non-null event $B \in \mathcal{F}$.

Definition: $\sigma(\Pi)$-consequentialism. For all Savage acts $f, g, h, h^{\prime}, h^{\prime \prime}, h^{\prime \prime \prime}$ and all nonnull events $B \in \sigma(\Pi)$, the following condition holds for ex post preferences:

$$
f_{B} h \succeq_{B} g_{B} h^{\prime} \Leftrightarrow f_{B} h^{\prime \prime} \succeq_{B} g_{B} h^{\prime \prime \prime}
$$

The notion of consequentialism in the context of decision trees under risk goes back to Hammond (1989) and Machina (1989) whereby Burks (1977) already refers to the same concept as "invariance axiom" (see the formal definition in Burks 1977, p. 268). In words, (12) states that it should not matter for ex post preferences conditional on observation $B$ whatever consequences, governed either by $h, h^{\prime}, h^{\prime \prime}$, or $h^{\prime \prime \prime}$, might have happened outside of $B$.

Definition: $\sigma(\Pi)$-dynamic consistency. For all Savage acts $f, g, h$ and all non-null events $B \in \sigma(\Pi)$, the following condition holds for the relationship between ex ante and ex post preferences:

$$
f_{B} h \succeq g_{B} h \Leftrightarrow f_{B} h \succeq_{B} g_{B} h
$$

Observation 2. $\sigma(\Pi)$-consequentialism (12) combined with $\sigma(\Pi)$-dynamic consistency (13) implies the $\sigma(\Pi)$-sure thing principle (11). 
Proof: Consider any non-null $B \in \sigma(\Pi)$. Observe that

$$
\begin{aligned}
f_{B} h & \succeq g_{B} h \\
& \Rightarrow f_{B} h \succeq_{B} g_{B} h \text { by }(13) \\
& \Rightarrow f_{B} h^{\prime} \succeq_{B} g_{B} h^{\prime} \text { by }(12) \\
& \Rightarrow f_{B} h^{\prime} \succeq_{B} h^{\prime} \text { by }(13),
\end{aligned}
$$

which proves the claim.

Definition: The universalism principle states that $\sigma(\Pi)=\mathcal{F}$.

Combined with Observation 2 the universalism principle implies that Savage's sure thing principle holds at all non-null events so that consequentialism, dynamic consistency and universalism give rise - under the remaining Savage axioms - to standard EU theory. ${ }^{8}$ Sarin and Wakker (1998b, Theorem 2.1) give up universalism. They show that updating within the fixed information structure of a given decision tree satisfies consequentialism and dynamic consistency whenever the set of multiple priors is given as the reduced family of probability measures. This reduced family of probability measures coincides with rectangular priors in the terminology of Epstein and Schneider (2003) who also fix some information structure $\Pi$ and assume that Savage's sure thing principle holds at all observable events in $\sigma(\Pi)$ only. As one implication of the rectangular multiple priors model, agents with different ex post information partitions must have different sets of priors, i.e., different ex ante ambiguity attitudes. As another implication, an agent who might learn in the future any possible information can not express any ambiguity attitudes under the rectangular multiple priors approach.

In our opinion, the rectangular MP approach demonstrates that the existence of ambiguity attitudes can only be reconciled with dynamic consistency under rather restrictive conditions. Hansen et al. (2006) argue along similar lines:

"If multiple priors truly are a statement of a decision maker's subjective beliefs, we think it is not appropriate to dismiss such beliefs on the grounds of dynamic inconsistency. Repairing that inconsistency through the enlargements necessary to induce rectangularity reduces the content of the original set of prior beliefs. In our context, this enlargement is immense, too immense to be interesting to us." (p. 78)

\footnotetext{
${ }^{8}$ This finding restates an implication of Lemma 2 in Ghirardato (2002) for our slightly different definitions of consequentialism and dynamic consistency.
} 


\subsection{Bayesian updating of non-additive probability measures}

A Bayesian update rule specifies how ex ante preference ordering $\succeq$ determines, for all non-null $B \in \mathcal{F}$, ex post preference ordering $\succeq_{B}$. In what follows we restrict attention to $(B, h)$-Bayesian update rules in the sense that there exists for every non-null $B \in \mathcal{F}$ and every pair of Savage acts $f, g$ some Savage act $h$, possibly depending on event $B$, such that

$$
f_{B} h \succeq g_{B} h \text { implies } f_{B} h^{\prime} \succeq_{B} g_{B} h^{\prime \prime} \text { for all } h^{\prime}, h^{\prime \prime}
$$

Such update rules obviously satisfy consequentialism since ex post preferences are independent of consequences that happened outside of observation $B .^{9}$

In case the sure thing principle is satisfied, the specification of $h$ in (14) would not matter for deriving ex post preferences. In case of CEU preferences, however, different specifications of $h$ in (14) result in different ways of updating ex ante CEU into ex post $\mathrm{CEU}$ preferences. Hence, several perceivable ways exist for defining a conditional capacity $\nu(\cdot \mid B)$ such that

$$
f \succeq_{B} g \Leftrightarrow E[U(f), \nu(\cdot \mid B)] \geq E[U(g), \nu(\cdot \mid B)]
$$

even if we restrict attention to consequentialist Bayesian update rules. For example, Gilboa and Schmeidler (1993) consider the benchmark cases of optimistic and pessimistic update rules. In these $h$ corresponds to the constant act giving the worst, respectively best, possible consequence. Gilboa and Schmeidler (1993) offer the following psychological motivation for the optimistic update rule:

"[...] when comparing two actions given a certain event $B$, the decision maker implicitly assumes that had $B$ not occurred, the worst possible outcome $[. .$.$] would have resulted.$ In other words, the behavior given $B[\ldots]$ exhibits 'happiness' that $B$ has occurred; the decisions are made as if we are always in 'the best of all possible worlds'." (p. 41)

Similarly, for the pessimistic update rule:

"[...] we consider a 'pessimistic' decision maker, whose choices reveal the hidden assumption that all the impossible worlds are the best conceivable ones." (p. 41f.)

\footnotetext{
${ }^{9}$ For an update rule for non-additive probability measures that violates consequentialism but satisfies dynamic consistency see Sarin and Wakker (1998a).
} 
The corresponding formal definitions of conditional capacities are given as

$$
\nu_{\text {opt }}(A \mid B)=\frac{\nu(A \cap B)}{\nu(B)} .
$$

for the optimistic and

$$
\nu_{\text {pess }}(A \mid B)=\frac{\nu(A \cup \neg B)-\nu(\neg B)}{1-\nu(\neg B)} .
$$

for the pessimistic update rule.

We further consider the full (or generalized) Bayesian update rule

$$
\nu_{F B}(A \mid B)=\frac{\nu(A \cap B)}{\nu(A \cap B)+1-\nu(A \cup \neg B)},
$$

which results when $h$ in (14) is given as the so-called conditional certainty equivalent of $g$ on $B$, i.e., $h$ is the constant act such that $g \sim_{B} h$ (Eichberger, Grant, and Kelsey 2006). Compared to (18) and (19), the full Bayesian update rule is less extreme and it performs empirically rather well, see, e.g., Cohen, Gilboa, Jaffray, and Schmeidler (2000) as well as Ludwig and Zimper (2011). A formal proof of the following observation appears in Zimper and Ludwig (2009).

\section{Observation 3. Bayesian updating of neo-additive capacities.}

1. An application of the optimistic update rule (15) to a neo-additive capacity (7) results in the conditional neo-additive capacity

$$
\nu_{\text {opt }}(A \mid B)=\delta_{\text {opt }}(B)+\left(1-\delta_{\text {opt }}(B)\right) \cdot \pi(A \mid B)
$$

with

$$
\delta_{\text {opt }}(B)=\frac{\delta \cdot \lambda}{\delta \cdot \lambda+(1-\delta) \cdot \pi(B)}
$$

2. An application of the pessimistic update rule (16) to a neo-additive capacity (7) results in the conditional neo-additive capacity

$$
\nu_{\text {pess }}(A \mid B)=\left(1-\delta_{\text {pess }}(B)\right) \cdot \pi(A \mid B)
$$

with

$$
\delta_{\text {pess }}(B)=\frac{\delta \cdot(1-\lambda)}{\delta \cdot(1-\lambda)+(1-\delta) \cdot \pi(B)} .
$$


3. An application of the full Bayesian update rule (17) to a neo-additive capacity (7) results in the conditional neo-additive capacity

$$
\nu_{F B}(A \mid B)=\delta_{F B}(B) \cdot \lambda+\left(1-\delta_{F B}(B)\right) \cdot \pi(A \mid B)
$$

with

$$
\delta_{F B}(B)=\frac{\delta}{\delta+(1-\delta) \cdot \pi(B)}
$$

\section{The behavioral model}

Based on Choquet decision theory we develop a behavioral model according to which agents' belief generation process is prone to a confirmatory bias heuristic whenever the asset's performance in terms of dividends is regarded as good, respectively bad. This performancedriven confirmatory bias - formally introduced in Subsection 3.2-implies dynamically inconsistent behavior. In Subsection 3.3 we formalize our assumption of sequential naïvety in the sense that agents are not aware of their confirmatory bias.

\subsection{Information structure}

Let $\left(Y_{t}\right)_{t>0}$ denote a sequence of random variables. The range of $Y_{t}$ is given by some finite set $\mathbf{Y}_{t} \subset \mathbb{R}_{+}$containing at least two elements. We interpret $Y_{t}$ as the asset's random period $t$ dividend payments which we identify - by convention - with the underlying firm's period $t$ profits. For our purpose it is convenient to consider $Y_{t}$ as coordinate variable on state space

$$
\Omega=\times_{t=0}^{\infty} \mathbf{Y}_{t},
$$

i.e., for all $t \geq 0, Y_{t}: \Omega \rightarrow \mathbf{Y}_{t}$ such that

$$
Y_{t}(\omega)=y_{t} \text { for } \omega=\left(y_{0}, y_{1}, \ldots\right) \text {. }
$$

As period t's agent information partition we define

$$
\mathcal{P}_{t}=\left\{\left\{\left(y_{0}, \ldots, y_{t}\right)\right\} \times\left(\times_{s=t+1}^{\infty} \mathbf{Y}_{t}\right)\right\}_{\left(y_{0}, \ldots, y_{t}\right) \in \times_{s=0}^{t} \mathbf{Y}_{j}}
$$

Observe that agents' information partitions get finer with increasing $t$. This implies that period $t$ agent can observe any realized stream of past dividend payments $y_{0}, \ldots, y_{t}$. Further denote by $\mathcal{F}_{t}$ the Borel $\sigma$-algebra generated by $\mathcal{P}_{t}$; that is, $\mathcal{F}_{t}$ contains all sets

$$
A \times\left(\times_{s=t+1}^{\infty} \mathbf{Y}_{s}\right)
$$


in $\Omega$ such that $A$ is any subset of $\times_{s=0}^{t} \mathbf{Y}_{s}$. As in standard infinite horizon asset pricing models we thus assume perfect memory since $\sigma$-algebras $\mathcal{F}_{t}$ generated by $\mathcal{P}_{t}$ constitute a filtration, i.e., $\mathcal{F}_{1} \subseteq \mathcal{F}_{2} \subseteq \ldots \subseteq \mathcal{F}$ whereby $\mathcal{F}$ denotes the standard product algebra generated by $\mathcal{F}_{1}, \mathcal{F}_{2}, \ldots$

\subsection{Performance-driven confirmatory bias heuristic}

Given a measurable space $(\Omega, \mathcal{F})$ we now describe agents' belief generation process by some conditional neo-additive probability measure $\nu(\cdot \mid \cdot)$. Recall that information received by a period $t$ agent is given as the stream of realizations of past profits $y_{0}, \ldots, y_{t}$. Let us formally define this information as the following event in $\mathcal{F}_{t}$

$$
I_{t}=\left(y_{0}, \ldots, y_{t}\right) \times\left(\times_{s=t+1}^{\infty} \mathbf{Y}_{s}\right)
$$

whereby we will henceforth speak of "agent $I_{t}$ " whenever a period $t$ agent has received information $I_{t}$.

Key to our behavioral approach is the heuristic assumption according to which period $t$ agent might have an optimistic, respectively pessimistic, confirmatory bias in the evaluation of an asset's future performance. This results from the agent's observation of the asset's past "performance". In line with most of the literature, we impose the simplifying-but plausible -assumption that an agent evaluates the asset's performance in terms of profits which coincide with dividend payments. More specifically, we interpret "good news in period $t$ " as the event of a dividend payment increase, i.e., $G_{t} \equiv\left(y_{t-1}<y_{t}\right)$, and "bad news in period $t$ " as a dividend payment decrease, i.e., $B_{t} \equiv\left(y_{t-1}>y_{t}\right)$. Denote by $j=k+1$ the number of good news (i.e., dividend increases), resp. bad news (i.e., dividend decreases), so that

$$
\begin{aligned}
\left(B_{t-k}, G_{t-k+1}, \ldots, G_{t}\right) & \equiv\left(y_{t-j-1}>y_{t-j}<\ldots<y_{t}\right) \\
\left(G_{t-k}, B_{t-k+1}, \ldots, B_{t}\right) & \equiv\left(y_{t-j-1}<y_{t-j}>\ldots>y_{t}\right) .
\end{aligned}
$$

The following assumptions on conditional neo-additive probability measure $\nu(\cdot \mid \cdot)$ naturally link our heuristic concept of "good" versus "bad" asset performance to optimistic versus pessimistic Bayesian update rules for neo-additive beliefs of Subsection 2.3. The so-called "switching" parameter, denoted $n$, thereby determines after how many good, respectively bad, news the agent switches to the optimistic, respectively pessimistic, updating rule. 
Confirmatory bias heuristic. Fix switching parameter $n \geq 1$ and let

$$
I_{t}=\left(y_{0}, \ldots, y_{t-n}, \ldots, y_{t}\right) \times\left(\times_{s=t+1}^{\infty} \mathbf{Y}_{s}\right) \in \mathcal{F}
$$

Then $\nu(\cdot \mid \cdot)$ must satisfy the following conditions.

(i) If $y_{t-n}<\ldots<y_{t}$, then agent $I_{t}$ is optimistically biased in the sense that, for all $A \in \mathcal{F}$,

$$
\nu\left(A \mid I_{t}\right)=\nu_{\text {opt }}\left(A \mid I_{t}\right)
$$

with $\nu_{\text {opt }}\left(A \mid I_{t}\right)$ given by (18).

(ii) If $y_{t-n}>\ldots>y_{t}$, then agent $I_{t}$ is pessimistically biased in the sense that, for all $A \in \mathcal{F}$,

$$
\nu\left(A \mid I_{t}\right)=\nu_{\text {pess }}\left(A \mid I_{t}\right)
$$

with $\nu_{\text {pess }}\left(A \mid I_{t}\right)$ given by (19).

(iii) If neither $y_{t-n}<\ldots<y_{t}$ nor $y_{t-n}>\ldots>y_{t}$, then, for all $A \in \mathcal{F}$,

$$
\nu\left(A \mid I_{t}\right)=\nu_{F B}\left(A \mid I_{t}\right)
$$

with $\nu_{F B}\left(A \mid I_{t}\right)$ given by (20).

Thereby, if the period $t$ agent has observed strictly increasing past profits $y_{t-n}, \ldots, y_{t}$ for a fixed number $n$ of periods, he interprets the prospect of future profits in an optimistic way. If, in contrast, he has observed strictly decreasing past profits $y_{t-n}, \ldots, y_{t}$, he interprets the prospect of future profits in a rather pessimistic way.

These heuristic assumptions on $\nu(\cdot \mid \cdot)$ conclude construction of $(\nu(\cdot \mid \cdot), \Omega, \mathcal{F})$ as conditional neo-additive probability space that governs agents' actual belief generation process about dividend payments.

\subsection{Sequentially naïve agents}

While $(\nu(\cdot \mid \cdot), \Omega, \mathcal{F})$ describes - by definition - the belief generation process for every agent $I_{t}$ about next period's profits $Y_{t+1}$, agents themselves have an incorrect view of this belief generation process. More precisely, we assume that every agent $I_{t}$ is convinced that his and his future selves' beliefs are governed by some process $\left(\nu^{I_{t}}(\cdot \mid \cdot), \Omega, \mathcal{F}\right)$ whereby $\nu\left(\cdot \mid I_{s}\right)$ and $\nu^{I_{t}}\left(\cdot \mid I_{s}\right)$ coincide whenever $s=t$ but not for future periods $s>t$. That is, our agents 
are "naïve" in the sense that they do not correctly anticipate that their future selves' beliefs are governed by $\nu(\cdot \mid \cdot)$ and not by $\nu^{I_{t}}(\cdot \mid \cdot)$.

In what follows we impose formal properties on $\nu^{I_{t}}(\cdot \mid \cdot)$ whereby we slightly misuse notation and simply write $y_{s+1}$ for event

$$
\left(Y_{s+1}=y_{s+1}\right) \in \mathcal{F}_{s+1}
$$

and $y_{0}, \ldots, y_{s}$ for event

$$
\left(Y_{0}=y_{0}, \ldots, Y_{s}=y_{s}\right) \in \mathcal{F}_{s}
$$

Agent $I_{t}$ 's "naïve" belief $\nu^{I_{t}}(\cdot \mid \cdot)$.

(i) Suppose that $I_{t}$ is optimistically biased. Then, for all $y_{s+1} \in \mathcal{F}_{s+1}$ and all $y_{0}, \ldots, y_{s} \in \mathcal{F}_{s}$ with $s \geq t$,

$$
\nu_{o p t}^{I_{t}}\left(y_{s+1} \mid y_{0}, \ldots, y_{s}\right)=\delta_{o p t}^{I_{t}}+\left(1-\delta_{o p t}^{I_{t}}\right) \cdot \pi\left(y_{s+1} \mid y_{0}, \ldots, y_{s}\right)
$$

whereby

$$
\delta_{o p t}^{I_{t}}=\frac{\delta \cdot \lambda}{\delta \cdot \lambda+(1-\delta) \cdot \pi\left(I_{t}\right)} .
$$

(ii) Suppose that $I_{t}$ is pessimistically biased. Then, for all $y_{s+1} \in \mathcal{F}_{s+1}$ and all $y_{0}, \ldots, y_{s} \in \mathcal{F}_{s}$ with $s \geq t$,

$$
\nu_{\text {pess }}^{I_{t}}\left(y_{s+1} \mid y_{0}, \ldots, y_{s}\right)=\left(1-\delta_{\text {pess }}^{I_{t}}\right) \cdot \pi\left(y_{s+1} \mid y_{0}, \ldots, y_{s}\right)
$$

whereby

$$
\delta_{\text {pess }}^{I_{t}}=\frac{\delta \cdot(1-\lambda)}{\delta \cdot(1-\lambda)+(1-\delta) \cdot \pi\left(I_{t}\right)} .
$$

(iii) Suppose that $I_{t}$ is neither optimistically nor pessimistically biased. Then, for all $y_{s+1} \in \mathcal{F}_{s+1}$ and all $y_{0}, \ldots, y_{s} \in \mathcal{F}_{s}$ with $s \geq t$,

$$
\nu_{F B}^{I_{t}}\left(y_{s+1} \mid y_{0}, \ldots, y_{s}\right)=\delta_{F B}^{I_{t}} \cdot \lambda+\left(1-\delta_{F B}^{I_{t}}\right) \cdot \pi\left(y_{s+1} \mid y_{0}, \ldots, y_{s}\right)
$$

whereby

$$
\delta_{F B}^{I_{t}}=\frac{\delta}{\delta+(1-\delta) \cdot \pi\left(I_{t}\right)}
$$


Whenever there exist ambiguity in our model, i.e., $\delta \neq 0$, our confirmatory bias heuristic gives rise to dynamic inconsistency. To see this for, e.g., two optimistically biased agents $I_{t}$ and $I_{s}$ such that $s>t$ observe that

$$
\begin{aligned}
\nu^{I_{t}}\left(y_{s+1} \mid y_{0}, \ldots, y_{s}\right) & =\delta_{o p t}^{I_{t}}+\left(1-\delta_{o p t}^{I_{t}}\right) \cdot \pi\left(y_{s+1} \mid y_{0}, \ldots, y_{s}\right) \\
& \neq \delta_{o p t}^{I_{s}}+\left(1-\delta_{o p t}^{I_{s}}\right) \cdot \pi\left(y_{s+1} \mid y_{0}, \ldots, y_{s}\right) \\
& =\nu^{I_{s}}\left(y_{s+1} \mid y_{0}, \ldots, y_{s}\right)=\nu\left(y_{s+1} \mid I_{s}\right)
\end{aligned}
$$

since $\delta_{\text {opt }}^{I_{t}} \neq \delta_{\text {opt }}^{I_{s}}$ because of $\pi\left(I_{t}\right)>\pi\left(I_{s}\right)$. That is, period $t$ agent $I_{t}$ bases his optimal action in period $s$-contingent on occurrence of observation $y_{0}, \ldots, y_{s}$ - on belief $\nu^{I_{t}}\left(\cdot \mid y_{0}, \ldots, y_{s}\right)$. This is different from belief $\nu^{I_{s}}\left(y_{s+1} \mid y_{0}, \ldots, y_{s}\right)$ of period $s$ agent who has actually observed $y_{0}, \ldots, y_{s}$. Hence, a period $t$ agent comes up with a contingent plan of future actions that are optimal from his period $t$ perspective, i.e., with respect to belief $\nu^{I_{t}}\left(\cdot \mid y_{0}, \ldots, y_{s}\right)$. However, this plan may be sub-optimal from the same agent's period $s>t$ perspective, i.e., with respect to belief $\nu^{I_{s}}\left(y_{s+1} \mid y_{0}, \ldots, y_{s}\right)$. As a consequence, there is a conflict of interest between an agent and his future selves.

By our assumption of sequentially naïve agents, we stipulate that agents are not aware of this conflict. Our agents are thus not sophisticated in the sense of Strotz (1956) and Pollak (1968) nor behavioral consistent in the sense of Karni and Safra (1990) nor sequentially rational in the sense of Kreps and Wilson (1982). Such sequentially rational agents would correctly anticipate dynamically inconsistent behavior of their future selves and accordingly incorporate it into their decisions. This is not the case for our agents who wrongly believe that their future selves will stick to the ex ante optimal plan of actions.

\section{The equilibrium price process}

In this section we derive corresponding equilibrium prices of our sequentially naïve representative agent economy as an adapted stochastic process $\left(p_{t}^{*}\right)_{t \geq 0}$ that consists of three different pricing regimes.

Consider the decision situation of a representative agent $I_{t}$ who maximizes his Choquet expected utility from an infinite consumption stream with respect to his belief $\nu^{I_{t}}(\cdot \mid \cdot)$ by deciding about his period $t, t+1, \ldots$ asset holdings. In line with most literature we assume that agent's vNM utility of an infinite consumption stream is additively time-separable.

Let $\beta \in(0,1)$ denote the time-discount factor and let $u: \mathbb{R} \rightarrow \mathbb{R}$ be a strictly concave, differentiable per period utility function. Denote by $z_{s} \in Z$, the agent's asset holdings in 
period $s$ for some open, bounded and convex $Z \subset \mathbb{R}_{+}$with $1 \in Z$. We set for the initial asset endowment $z_{0}=1$. Let $p_{s}$ denote the period $s$ asset price. The agent's maximization problem is then given as

$$
\max _{\left(z_{t}, z_{t+1}, \ldots\right)} u\left(c_{t}\right)+E\left[\sum_{s=t+1}^{\infty} \beta^{s-t} u\left(c_{s}\right), \nu^{I_{t}}(\cdot \mid \cdot)\right]
$$

subject to

$$
c_{s}=Y_{s} z_{s}+p_{s}\left(z_{s}-z_{s+1}\right) \text { for all } s \geq t .
$$

In our representative agent economy it must hold for any period $t$ equilibrium allocation that $z_{t}^{*}=1$. We further assume that Choquet expected utility

$$
E\left[\sum_{s=t+1}^{\infty} \beta^{s-t} u\left(c_{s}\right), \nu^{I_{t}}(\cdot \mid \cdot)\right]
$$

is finite for all $\left(z_{t}, z_{t+1}, \ldots\right)$. Define for $s>t$ the following random variable (the stochastic discount factor)

$$
M_{t, s}=\beta^{s-t} \cdot \frac{u^{\prime}\left(c_{s}\right)}{u^{\prime}\left(c_{t}\right)} .
$$

Consider now the special case of an expected utility decision maker where $\nu$ degenerates to the "true" probability measure $\pi$. In this degenerate case the period $t$ asset price is given by the standard formula

$$
p_{t}^{E U}=E\left[\sum_{s=t+1}^{\infty} M_{t, s} \cdot Y_{s}, \pi\left(y_{s}\right)\right] .
$$

In contrast to this degenerate case, however, an ambiguous belief $\nu$ gives rise to an equilibrium price process $\left(p_{t}^{*}\right)_{t \geq 0}$ where we have to consider three different pricing regimes. These regimes reflect the three possibilities that agent $I_{t}$ either has an optimistic, or a pessimistic or no confirmatory bias.

Equilibrium pricing scheme. Fix switching parameter $n \geq 1$. For all $t \geq 0$, the period $t$ equilibrium price is determined as follows

$$
p_{t}^{*}= \begin{cases}p_{t}^{o p t} & \text { if } y_{t-n}<\ldots<y_{t}, \\ p_{t}^{\text {pess }} & \text { if } y_{t-n}>\ldots>y_{t}, \\ p_{t}^{F B} & \text { else. }\end{cases}
$$

The following findings establish for all three pricing regimes the existence of unique period $t$ equilibrium prices $p_{t}^{o p t}, p_{t}^{p e s s}$, and $p_{t}^{F B}$ conditional on received information $I_{t}=$ $\left(y_{0}, . ., y_{t}\right)$. A formal derivation of these findings in done in Appendix A.1. 
"Optimistically biased pricing regime". Suppose that agent $I_{t}$ is optimistically biased. Then there exists a unique period $t$ equilibrium asset price such that

$$
p_{t}^{o p t}=\delta_{o p t}^{I_{t}} \cdot \sum_{s=t+1}^{\infty} \min M_{t, s} \cdot \max Y_{s}+\left(1-\delta_{o p t}^{I_{t}}\right) \cdot p_{t}^{E U}
$$

such that

$$
\delta_{o p t}^{I_{t}}=\frac{\delta \cdot \lambda}{\delta \cdot \lambda+(1-\delta) \cdot \pi\left(I_{t}\right)}
$$

whenever the transversality condition

$$
\lim _{s \rightarrow \infty}\left(M_{t, s} \cdot p_{s}^{o p t}\right)=0
$$

holds.

"Pessimistically biased pricing regime". Suppose that agent $I_{t}$ is pessimistically biased. Then there exists a unique period $t$ equilibrium asset price such that

$$
\begin{aligned}
p_{t}^{\text {pess }}= & \delta_{\text {pess }}^{I_{t}} \cdot \sum_{s=t+1}^{\infty} \max M_{t, s} \cdot \min Y_{s} \\
& +\left(1-\delta_{\text {pess }}^{I_{t}}\right) \cdot p_{t}^{E U}
\end{aligned}
$$

such that

$$
\delta_{\text {pess }}^{I_{t}}=\frac{\delta \cdot(1-\lambda)}{\delta \cdot(1-\lambda)+(1-\delta) \cdot \pi\left(I_{t}\right)} .
$$

whenever the transversality condition

$$
\lim _{s \rightarrow \infty}\left(M_{t, s} \cdot p_{s}^{p e s s}\right)=0
$$

holds.

"Full Bayesian pricing regime". Suppose that agent $I_{t}$ is neither optimistically nor pessimistically biased. Then there exists a unique period $t$ equilibrium asset price such that

$$
\begin{aligned}
p_{t}^{F B}= & \delta_{F B}^{I_{t}} \cdot\left(\lambda \sum_{s=t+1}^{\infty} \min M_{t, s} \cdot \max Y_{s}+(1-\lambda) \sum_{s=t+1}^{\infty} \max M_{t, s} \cdot \min Y_{s}\right) \\
& +\left(1-\delta_{F B}^{I_{t}}\right) \cdot p_{t}^{E U}
\end{aligned}
$$


such that

$$
\delta_{F B}^{I_{t}}=\frac{\delta}{\delta+(1-\delta) \cdot \pi\left(I_{t}\right)} .
$$

whenever the transversality condition

$$
\lim _{s \rightarrow \infty}\left(M_{t, s} \cdot p_{s}^{F B}\right)=0
$$

holds.

Under the assumption that the subjective additive measure $\pi$ coincides with the objective probability measure, we can think of (21) as the "correct" price. This properly takes into account the stochastic process of economic fundamentals as well as the representative agent's "tastes" for intertemporal consumption smoothing as expressed by the stochastic discount factor. For almost risk-neutral agents, whose stochastic discount factor, $M_{t, s}$, is approximately given by time-discount factor $\beta^{s-t}$, we readily obtain the following result.

Observation 4. Consider (almost) risk-neutral agents with ambiguity parameter $\delta>0$.

(i) The optimistic pricing regime always overprices the asset in the sense that $p_{t}^{\text {opt }}>$ $p_{t}^{E U}$.

(ii) The pessimistic pricing regime always underprices the asset in the sense that $p_{t}^{\text {pess }}<p_{t}^{E U}$.

(iii) The full Bayesian pricing regime overprices the asset iff

$$
\sum_{s=t+1}^{\infty} \beta^{s-t} \cdot\left(\lambda \max Y_{s}+(1-\lambda) \cdot \min Y_{s}\right)>\sum_{s=t+1}^{\infty} \beta^{s-t} \cdot E\left[Y_{s}, \pi\right]
$$

whereby

$$
p_{t}^{\text {pess }}<p_{t}^{F B}<p_{t}^{o p t}
$$

\section{Underreaction and overreaction revisited}

Rewriting the formal definitions of underreaction (2) and overreaction (3) in terms of dividend increases, respectively decreases, gives: 
- Underreaction to a sequence of $j \geq 1$ good news:

$$
E\left[R_{t+1}, \pi\left(\cdot \mid y_{t-j-1}>y_{t-j}<\ldots<y_{t}\right)\right]>E\left[R_{t+1}, \pi\left(\cdot \mid y_{t-j-1}<y_{t-j}>\ldots>y_{t}\right)\right] .
$$

- Overreaction to a sequence of $j \geq 1$ good news:

$$
E\left[R_{t+1}, \pi\left(\cdot \mid y_{t-j-1}>y_{t-j}<\ldots<y_{t}\right)\right]<E\left[R_{t+1}, \pi\left(\cdot \mid y_{t-j-1}<y_{t-j}>\ldots>y_{t}\right)\right] .
$$

For illustrative reasons we assume that the subjective additive part of the agents' neoadditive capacity, i.e., $\pi$, coincides with the "objective" probability measure that governs the dividend payment process $\left(Y_{t}\right)_{t \geq 0}$. We further assume that the $Y_{t} \in\{\min Y, \ldots, \max Y\}$ are i.i.d. and that $\pi$ has full support on $\{\min Y, \ldots, \max Y\}$. The following Proposition (proved in detail in Appendix A.2) comprehensively describes the conditions under which our model generates underreaction, respectively overreaction, for any given probability measure $\pi$.

Proposition. Consider (almost) risk-neutral agents with ambiguity parameter $\delta>0$ and fix switching parameter $n \geq 1$.

(i) Let $j<n-1$. Then

$$
E\left[R_{t+1}, \pi\left(\cdot \mid y_{t-j-1}>y_{t-j}<\ldots<y_{t}\right)\right]=E\left[R_{t+1}, \pi\left(\cdot \mid y_{t-j-1}<y_{t-j}>\ldots>y_{t}\right)\right] .
$$

(ii) Let $j=n-1$. Then underreaction (24) holds for all histories such that

$$
\begin{aligned}
& \left(y_{t-j-1}>y_{t-j}<\ldots<y_{t}\right) \text { with } y_{t} \neq \max Y \text { or } \\
& \left(y_{t-j-1}<y_{t-j}>\ldots>y_{t}\right) \text { with } y_{t} \neq \min Y .
\end{aligned}
$$

For histories

$$
\begin{aligned}
& \left(y_{t-j-1}>y_{t-j}<\ldots<\max Y\right) \text { and } \\
& \left(y_{t-j-1}<y_{t-j}>\ldots>\min Y\right)
\end{aligned}
$$

we have instead

$$
E\left[R_{t+1}, \pi\left(\cdot \mid y_{t-j-1}>y_{t-j}<\ldots<\max Y\right)\right]=E\left[R_{t+1}, \pi\left(\cdot \mid y_{t-j-1}<y_{t-j}>\ldots>\min Y\right)\right] .
$$


(iii) Let $j \geq n$. Then overreaction (25) holds whenever $t$ is sufficiently large.

For i.i.d. dividend payments, expected returns remain constant across all possible dividend news as long as no change in the pricing regime is triggered in period $t+1$. By part (i) of the Proposition, this is the case in our model if the number of good (resp. bad) news that could be observed in period $t+1$ is less than the value of the switching parameter, i.e., if $j+1<n$. If the set of possible dividend payments $\{\min Y, \ldots, \max Y\}$ contains at least $n$ elements, histories like (27) will occur with strictly positive probability. As a consequence, there will be, by part (ii) of the Proposition, underreaction with probability one whenever the number of good (resp. bad) news that are possibly observable in period $t+1$ equals the value of the switching parameter, i.e., if $j+1=n$. Finally, by part (iii) of the Proposition, our model will always generate overreaction in case the number of good (resp. bad) news that are possibly observable in period $t+1$ is strictly greater than the value of the switching parameter, i.e., if $j+1>n$.

Observe that our model generates a very distinctive pattern of under- versus overreaction: For a strictly increasing number of good (resp. bad) news there will be eventually underreaction, immediately followed by overreaction if the number of good (resp. bad) news increases further. Consequently, any empirical return pattern (for i.i.d. dividend payments) that initially generates overreaction whereas it generates underreaction when the number of good (resp. bad) news increases is not compatible with our model. Turn now to the empirically predominant phenomena (4) and (5), discussed in Barberis et al. (1998), and rewrite them in terms of dividend changes as follows.

- Underreaction to a single period of good news:

$$
E\left[R_{t+1}, \pi\left(\cdot \mid y_{t-2}>y_{t-1}<y_{t}\right)\right]>E\left[R_{t+1}, \pi\left(\cdot \mid y_{t-2}<y_{t-1}>y_{t}\right)\right]
$$

- Overreaction to multiple periods of good news: For all $j \geq 2$,

$$
E\left[R_{t+1}, \pi\left(\cdot \mid y_{t-j-1}>y_{t-j}<\ldots<y_{t}\right)\right]<E\left[R_{t+1}, \pi\left(\cdot \mid y_{t-j-1}<y_{t-j}>\ldots>y_{t}\right)\right]
$$

By the Proposition, underreaction to a single period of good news, i.e., $j=1$, happens if and only if $n=2$. Furthermore, for $n=2$ part (iii) of the Proposition implies that overreaction to multiple periods of good news will occur for sufficiently large $t$. 
Corollary. Fix the assumptions of the Proposition. Our model simultaneously generates underreaction to a single period of good news as well as overreaction to multiple periods of good news (in the long run), if and only if, we have that the switching parameter is $n=2$.

Remark. Notice that the above results rely on the assumption of i.i.d. dividend payments. By this i.i.d. assumption, we can neglect for the inequalities (24) and (25) the expected dividend payments for period $t+1$ since we have for the lhs and rhs of both inequalities

$$
\begin{aligned}
E\left[Y_{t+1}, \pi\left(\cdot \mid y_{t-j-1}>y_{t-j}<\ldots<y_{t}\right)\right] & =E\left[Y_{t+1}, \pi\left(\cdot \mid y_{t-j-1}<y_{t-1}>\ldots>y_{t}\right)\right] \\
& =E\left[Y_{t+1}, \pi\right] .
\end{aligned}
$$

As a consequence, our findings on period $t+1$ returns are exclusively driven by properties of equilibrium prices $p_{t}^{*}, p_{t+1}^{*}$. The situation would be different-and technically more complex - if we give up the i.i.d. assumption. For example, if we consider a dividend process given as a symmetric random walk (as., e.g., in Barberis et al. 1998), we have, on average, that

$$
E\left[Y_{t+1}, \pi\left(\cdot \mid y_{t-j-1}>y_{t-j}<\ldots<y_{t}\right)\right]>E\left[Y_{t+1}, \pi\left(\cdot \mid y_{t-j-1}<y_{t-1}>\ldots>y_{t}\right)\right]
$$

because the expected value of next period dividend payments, being equal to the present period's dividend payment, will be on average higher for increasing than for decreasing sequences. But if (33) instead of (32) holds on average, this would imply for our model that overreaction becomes harder to establish whereas underreaction becomes easier to establish. We regard it as an interesting task for future research to look into possible generalizations of our results to stationary Markov dividend processes.

\section{A numerical illustration of equilibrium returns}

We now take a calibrated version of our model in order to illustrate price patterns generated by our model for different values of the confirmatory bias parameter $n$. In a next step, we report that simulated returns accord with the predictions of our proposition and corollary for different parameter constellations $n$ and $j$. As the model is highly stylized, our exercise is for illustrative purposes only and we do not attempt to provide a realistic parametrization. Throughout, we assume that agents are risk neutral and that dividends are i.i.d.. 


\subsection{Calibration}

We assume that dividends are distributed as i.i.d. normal with mean $E Y$ and variance $\sigma_{Y}^{2}$. We approximate this distribution by taking discrete realizations applying Gaussian quadrature methods using Chebychev nodes and weights. We set $E Y=1.5, \sigma_{Y}=0.1$, and take $m=11$ discrete realizations (nodes).

By the assumptions of risk-neutrality and i.i.d. dividends, the equilibrium price under rational expectations in equation (21) is given by

$$
p_{t}^{E U}=\sum_{s=t+1}^{\infty} \beta^{t-s} E Y=\frac{\beta}{1-\beta} E Y .
$$

Consequently, the expected return under rational expectations is

$$
E R_{t+1}^{E U}=1+E r_{t+1}^{E U}=E \frac{p_{t+1}^{E U}+Y_{t+1}}{p_{t}^{E U}}=\frac{1}{\beta} .
$$

We interpret the frequency of our model as annual and therefore consider as a realistic value for the real return on the risky asset under rational expectations $E r_{t+1}^{E U}=0.075$. With this target value, the implied value for the annual discount factor is

$$
\beta=\frac{1}{E R_{t+1}^{E U}}=0.93023
$$

and we therefore have that

$$
p_{t}^{E U}=\frac{\beta}{1-\beta} E Y=\frac{1}{E r_{t+1}^{E U}} E Y=20 .
$$

According to section 4, asset prices in the three pricing regimes according to our assumptions are given by

$$
p_{t}^{o p t}=\delta_{o p t}^{t} \cdot \frac{\beta}{1-\beta} \cdot \max Y+\left(1-\delta_{o p t}^{t}\right) \cdot p_{t}^{E U}
$$

with

$$
\delta_{o p t}^{t}=\frac{\delta \cdot \lambda}{\delta \cdot \lambda+(1-\delta) \cdot \prod_{s=0}^{t} \pi\left(y_{s}\right)},
$$

as well as

$$
p_{t}^{\text {pess }}=\delta_{\text {pess }}^{t} \cdot \frac{\beta}{1-\beta} \cdot \min Y+\left(1-\delta_{\text {pess }}^{t}\right) \cdot p_{t}^{E U}
$$

with

$$
\delta_{p e s s}^{t}=\frac{\delta \cdot(1-\lambda)}{\delta \cdot(1-\lambda)+(1-\delta) \cdot \prod_{s=0}^{t} \pi\left(y_{s}\right)}
$$


and

$$
p_{t}^{F B}=\delta_{F B}^{t} \cdot \frac{\beta}{1-\beta} \cdot(\lambda \cdot \max Y+(1-\lambda) \cdot \min Y)+\left(1-\delta_{F B}^{t}\right) \cdot p_{t}^{E U}
$$

with

$$
\delta_{F B}^{t}=\frac{\delta}{\delta+(1-\delta) \cdot \prod_{s=0}^{t} \pi\left(y_{s}\right)} .
$$

We focus our analysis on the stationary state of the model and set $\delta=\delta_{\infty}=1^{10}$ Corresponding equilibrium prices are:

$$
\begin{aligned}
p_{t}^{\text {opt }} & =\frac{\beta}{1-\beta} \cdot \max Y \\
p_{t}^{\text {pess }} & =\frac{\beta}{1-\beta} \cdot \min Y \\
p_{t}^{F B} & =\frac{\beta}{1-\beta} \cdot(\lambda \cdot \max Y+(1-\lambda) \cdot \min Y) .
\end{aligned}
$$

We determine the value of $\lambda$ such that in all periods $t$ the price under rational expectations is the same as in the full Bayesian pricing regime. This means that we rule out overpricing, respectively underpricing, in the full Bayesian pricing regime, cf. Equation (22). We accordingly solve

$$
p_{t}^{F B}=\delta_{F B}^{t} \cdot \frac{\beta}{1-\beta} \cdot(\min (Y)+\lambda(\max (Y)-\min (Y)))+\left(1-\delta_{t}^{F B}\right) p_{t}^{E U}=p_{t}^{E U}
$$

for $\lambda$ which, given that the distribution of dividends is symmetric, results in $\lambda=0.5$.

We consider four different scenarios for switches of pricing regimes according to our heuristic as described in subsection 3.2. Recall from these heuristics that, starting from the dividend payment in period $t-n, y_{t-n}$, it takes a sequence of length $n$ of increasing, respectively decreasing, dividend realizations to induce a shift to the optimistic, respectively pessimistic, pricing regime. We set $n \in\{1, \ldots, 3\}$. When evaluating the conditional return expressions in equation (24), respectively equation (25), numerically, we set $j \in\{1, \ldots, 4\}$. We then report results on returns for all combinations $n \otimes j$.

We take the number of periods to be $T=200$ and discard the first 99 observations. Throughout, we compute averages over $M=5000$ stochastic simulations.

All calibration parameters of our model are summarized in Table 1.

\footnotetext{
${ }^{10}$ As convergence of $\prod_{s=0}^{t} \pi\left(y_{s}\right) \rightarrow 0$ is fast, this would be reached in few periods only, even for very low levels of initial ambiguity, e.g., $\delta_{0}=1.0 E^{-10}$.
} 
Table 1: Calibration

\begin{tabular}{lc}
\hline \hline Expected value of dividends, $E Y$ & 1.5 \\
Standard Deviation of dividends, $\sigma_{Y}$ & 0.1 \\
Number of nodes, $m$ & 11 \\
Expected rate of return, $E r_{t+1}^{E U}$ & 0.075 \\
Discount factor, $\beta$ & 0.93023 \\
Degree of pessimism, $\lambda$ & 0.5 \\
Degree of ambiguity, $\delta$ & 1.0 \\
Confirmatory bias parameter, $n$ & $\{1, \ldots, 3\}$ \\
Sequence of good (bad) news, $j$ & $\{1, \ldots, 4\}$ \\
Time Horizon, $T$ & 200 \\
Stochastic Simulations, $M$ & 1000 \\
\hline \hline
\end{tabular}

\subsection{Results}

We start by an illustration of the time paths of key variables for the first stochastic simulation, $m=1$. Panel (a) of Figure 1 displays the random time path of dividends for periods $100-200$, the exogenous driving process of our model. In panel (b) of the same figure, we zoom in a selected period 180-185. In this period, we observe a path of increasing dividend realizations between $t=181$ and $t=184$.

We next analyze equilibrium prices and returns for this stochastic simulation. We focus on two scenarios where $n=2$, respectively $n=3$. Equilibrium asset returns in the three pricing regimes are shown, for the two alternative parameterizations of $n$, in figures $2-3$. In each figure, the rational expectations equilibrium return is the solid black line (respectively black squares). The actual equilibrium returns emanating in our model are shown as dashed blue lines (respectively blue diamonds). Furthermore, Table 2 provides summary information for selected time period $180-185$ and endogenous prices, prices plus dividends and returns in this period.

We start with the case where $n=3$, i.e., the series of dividend realizations $y_{t-3}>\ldots>$ $y_{t}$, respectively $y_{t-3}<\ldots<y_{t}$, induces a shift in $t$ to the optimistic, respectively pessimistic, pricing regime. For $n=3$ such a shift is very unlikely, and, as seen from Figure 2, only one occurs. As dividend payments monotonically increase from $t=181$ to $t=184$, cf. panel (b) in Figure 1, prices shift to the optimistic regime in $t=184$, cf. Table 2. As dividend 
Figure 1: Dividends

(a) Period 100-200

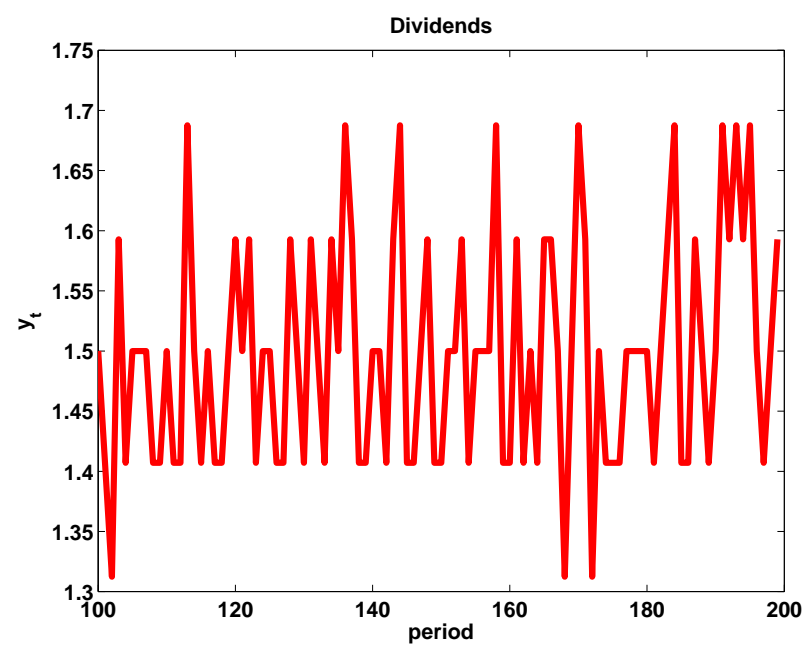

(b) Period 180-185

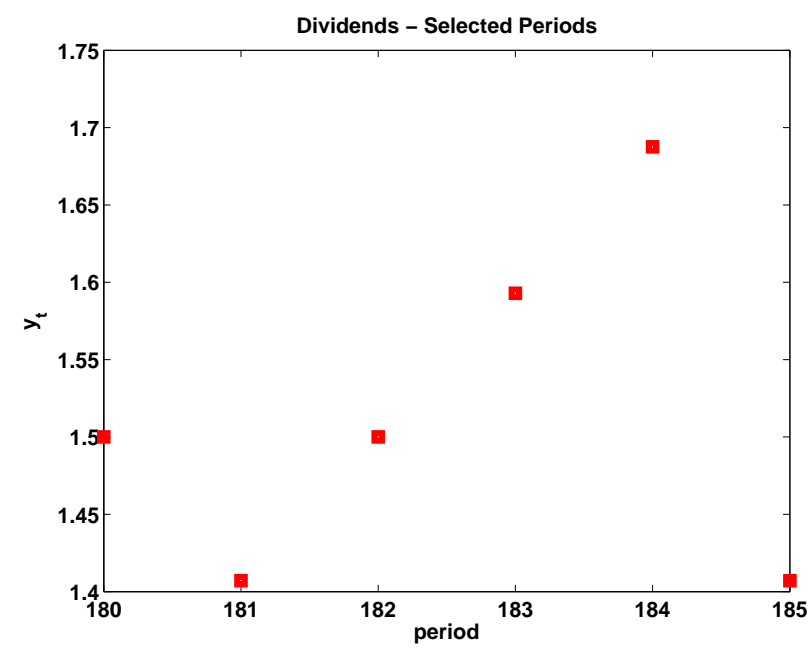

payments then decrease from $t=184$ to $t=185$, prices revert back to the full Bayesian pricing regime in $t=185$. Accordingly, the return in $t=184$ is above 40 percent and, as a consequence of trend reversal in prices, at below $-20 \%$ in $t=185$. Resulting returns in this subperiod are displayed in panel (b) of Figure 2.

In case that $n=2$, the series of dividend realizations $y_{t-2}>\ldots>y_{t}$, respectively $y_{t-2}<$ $\ldots<y_{t}$ induces a shift in $t$ to the optimistic, respectively pessimistic, pricing regime. Accordingly, shifts between regimes are more likely to occur and, during period 100 to 200, we observe 8 cases with optimistic and 9 cases with pessimistic pricing regimes, cf. panel (a) of Figure 3. Notice that this includes cases in which optimistic, respectively pessimistic, pricing regimes persist for at least two subsequent periods.

It is again instructive to more closely look at subperiod $180-185$, displayed in panel (b) of Figure 3. In comparison to the case where $n=3$, the shift to the optimistic pricing regime now already occurs in $t=183$, also see Table 2 . Accordingly, the return in $t=183$ jumps up to above 40 percent. In $t=184$, the optimistic pricing regime continues to be in place. Hence, the return moves down to roughly 6 percent which is lower than the return under rational expectations due to the higher base price in $t=183$. Finally, when prices revert to the full Bayesian regime $t=185$, the return is again less than 20 percent. 
Figure 2: Returns: $n=3$

(a) Period 100-200

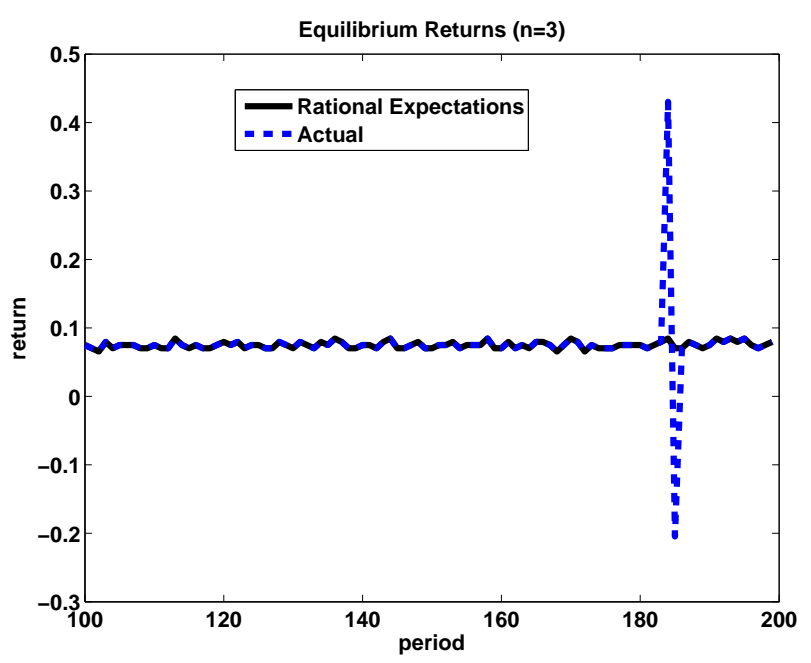

(b) Period 180-185

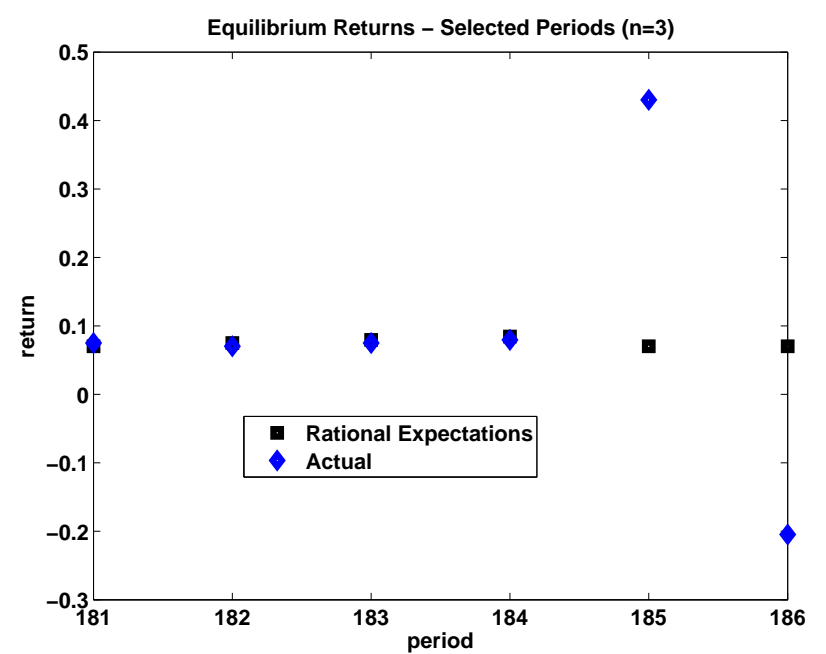

Figure 3: Returns: $n=2$

(a) Period 100-200

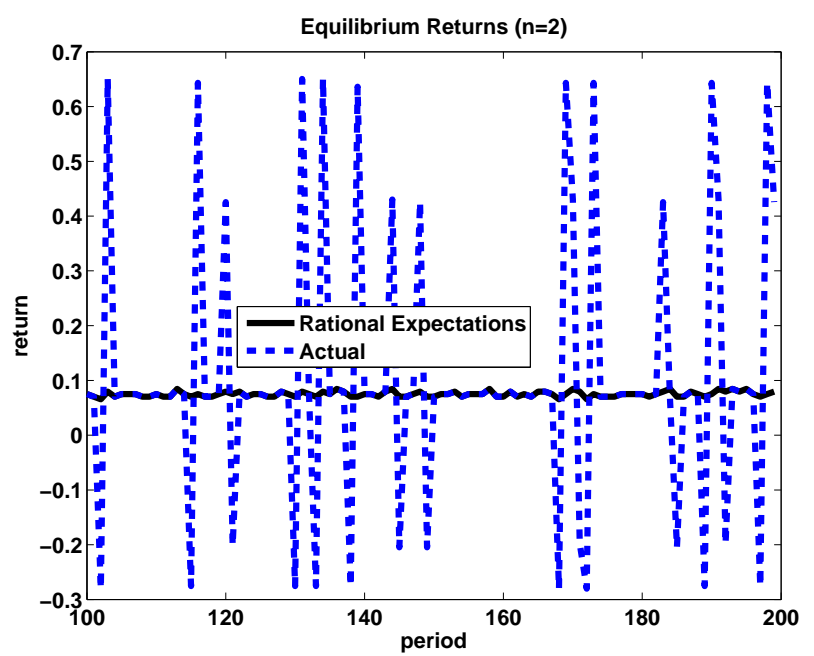

(b) Period 180-185

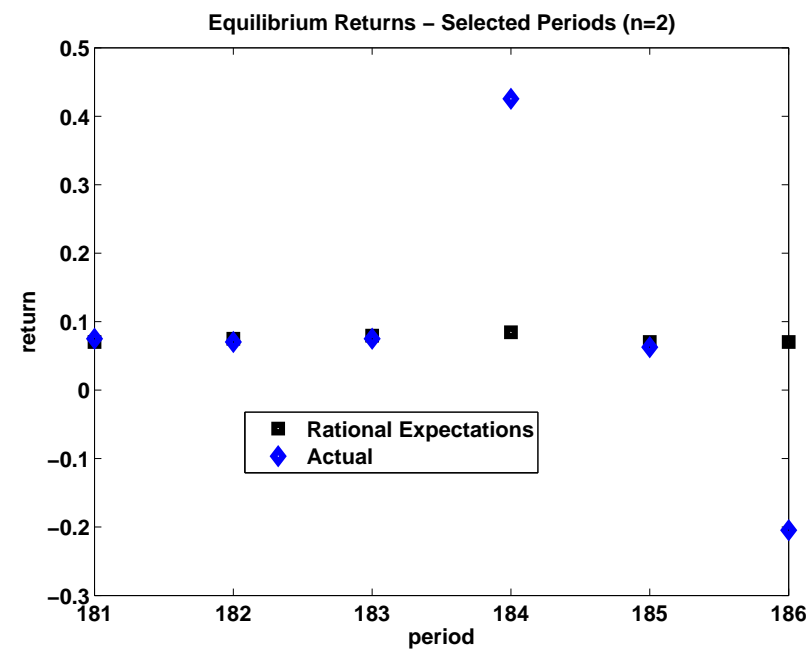


Table 2: Summary: Selected Periods

\begin{tabular}{lccc}
\hline \hline$t$ & R.E. & Actual $(\mathrm{n}=3)$ & Actual $(\mathrm{n}=2)$ \\
\hline 180 & 20.00 & 20.00 & 20.00 \\
181 & 20.00 & 20.00 & 20.00 \\
182 & 20.00 & 20.00 & 20.00 \\
183 & 20.00 & 20.00 & 26.92 \\
184 & 20.00 & 26.92 & 26.92 \\
185 & 20.00 & 20.00 & 20.00 \\
\hline & Prices plus Dividends \\
\hline 180 & 21.50 & 21.50 & 21.50 \\
181 & 21.41 & 21.41 & 21.41 \\
182 & 21.50 & 21.50 & 21.50 \\
183 & 21.59 & 21.59 & 28.51 \\
184 & 21.69 & 28.60 & 28.60 \\
185 & 21.41 & 21.41 & 21.41 \\
\hline & \multicolumn{3}{c}{ Returns } \\
\hline 181 & 0.0704 & 0.0704 & 0.0704 \\
182 & 0.0750 & 0.0750 & 0.0750 \\
183 & 0.0796 & 0.0796 & 0.4255 \\
184 & 0.0844 & 0.4302 & 0.0627 \\
185 & 0.0704 & -0.2047 & -0.2047 \\
186 & 0.0704 & 0.0704 & 0.0704 \\
\hline \hline
\end{tabular}


Finally, we evaluate conditional expected returns, cf. equation (24), respectively (25), by averaging across all 5000 simulation periods. We do so for all combinations of $n \in$ $\{1, \ldots, 3\}$ and $j \in\{1, \ldots, 4\}$. Results are summarized in Table 3. These confirm our Proposition and Corollary. First, turn to the case where $n=3, j=1$ for which we have $E\left[R_{t+1}, \pi\left(\cdot \mid y_{t-2}>y_{t-1}<y_{t}\right)\right]=E\left[R_{t+1}, \pi\left(\cdot \mid y_{t-2}<y_{t-1}>y_{t}\right)\right]$ (subject to a numerical tolerance set to $1.0 e-06)$, cf. Proposition (i). Second, observe that for all combinations $j \geq n$ we have overreaction, cf. Proposition (iii). Third, for $n=2$ we observe underreaction when $j=1$, as stated in Proposition (ii) and the Corollary. Fourth, underreaction is observed for $n=3$ only when $j=n-1=2$, cf. Proposition (ii) and the Corollary.

Table 3: Expected Returns

\begin{tabular}{lcc}
\hline \hline & $E\left[R_{t+1}, \pi\left(\cdot \mid y_{t-j-1}>y_{t-j}<\ldots<y_{t}\right)\right]$ & $E\left[R_{t+1}, \pi\left(\cdot \mid y_{t-j-1}<y_{t-j}>\ldots>y_{t}\right)\right]$ \\
\hline$n=1, j=1$ & -0.2803 & 0.8056 \\
$n=1, j=2$ & -0.3605 & 0.9711 \\
$n=1, j=3$ & -0.4107 & 1.0535 \\
$n=1, j=4$ & -0.4334 & 1.1355 \\
\hline$n=2, j=1$ & 0.1494 & 0.0004 \\
$n=2, j=2$ & -0.1778 & 0.5949 \\
$n=2, j=3$ & -0.1927 & 0.6195 \\
$n=2, j=4$ & -0.2011 & 0.6437 \\
\hline$n=3, j=1$ & 0.0750 & 0.0750 \\
$n=3, j=2$ & 0.1065 & 0.0433 \\
$n=3, j=3$ & -0.1927 & 0.6195 \\
$n=3, j=4$ & -0.2011 & 0.6437 \\
\hline \hline
\end{tabular}




\section{Related literature and critical discussion}

To model underreaction and overreaction, the existing literature considers three different scenarios with respect to the nature and reception of information about a firm's earnings. Daniel, Hirshleifer, and Subrahmanyam (1998) differentiate between public and private information whereby investors are assumed to believe too strongly in their own information and discount public signals. Consequently, there is underreaction to public information but overreaction to private information. Hong and Stein (1999) base their theory on the assumption that there exist heterogenous groups of investors which differ in their reactions to public news. In particular, one of these groups ignores news but reacts to prices only. As a consequence, there is initial underreaction followed by subsequent overreaction.

Most closely related to our own approach is Barberis, Shleifer and Vishny (1998) (BSV). BSV consider a representative investor whose beliefs deviate from rational expectations because of stylized psychological attitudes such as "conservatism" and "overconfidence". Formally, BSV describe the "true" dividend process as a symmetric random walk governed by objective probability measure $\pi$. As a deviation from the rational expectations paradigm, the representative investor bases his portfolio maximization problem on a subjective additive probability measure $\tilde{\pi} \neq \pi$ which is constructed in a highly non-stationary way. More specifically, BSV stipulate that the investor considers exactly two different regimes as possible - one regime with positive the other regime with negative correlation between today's and tomorrow's dividends. These two different stochastic processes are combined through subjective regime-switching probabilities into an aggregate stochastic process of dividend payments governed by subjective measure $\tilde{\pi}$. As their main formal result BSV prove - under specific parametric assumptions - that conditional subjective prob-

ability measures $\tilde{\pi}\left(\omega \mid y_{1}, \ldots, y_{t}\right)$ can generate underreaction and overreaction in the sense of (4) and (5).

On the one hand, the article by BSV is a highlight in the financial economics literature because it identifies and addresses in a very precise way empirical pricing phenomena that violate the EMH. On the other hand, however, the behavioral approach of BSV is quite ad hoc in that the formal construction of subjective probability measure $\tilde{\pi}$ directly aims at generating return patterns (4) and (5) whereby any psychological explanation of this specific formalism in terms of conservatism and representativeness remains vague - too vague for our taste. In contrast to BSV, our model is less ad-hoc. Our formalism is based on axiomatically founded Bayesian update rules that admit for straightforward psychological interpretation within the framework of Choquet decision theory. 
Our own behavioral ad hoc assumption is a plausible confirmatory bias heuristic. It links a sequence of good, respectively bad, news to the optimistic, respectively, pessimistic Bayesian update rule. Remaining sequences of news are updated through the full Bayesian update rule. Whether such an ad hoc link between good (bad) news and the optimistic (pessimistic) interpretation of such news is viable, is ultimately an empirical question which we want to address in future research. Nevertheless, the fact that this simple confirmatory bias heuristic is indeed able to generate the return patterns (4) and (5) came rather unexpected to us since - unlike BSV - nothing in our set-up has been intentionally "geared" towards the possible generation of under- and overreaction.

Also notice that our approach addresses an important self-criticism of BSV who remark: "The idea that the investor believes that the world is governed by one of the two incorrect models is a crude way of capturing the psychological phenomena of the previous section." (p. 318). While BSV thus introduce the inability of agents to learn the correct earnings process in the long run by assumption, our model gives rise to it by our description of agents' beliefs as non-additive probability measures. Further observe that our agent is a Bayesian decision maker in the sense that he holds a unique prior neo-additive belief defined over all possible future events which he updates through an application of Bayesian update rules. Zimper and Ludwig (2009) and Zimper (2009, 2011) also demonstrate that Bayesian learning of neo-additive beliefs with respect to update rules considered in this paper does, in general, not converge to correct (additive) probabilities when the agent observes arbitrarily large samples drawn from some i.i.d. process. As a consequence, our approach is not prone to the standard criticism against the relevance of incorrect beliefs stating that subjective beliefs of Bayesian learners will eventually converge to true probabilities. ${ }^{11}$

On purpose, we develop - by our restriction to neo-additive capacities - a parsimonious model and keep the analysis extremely stylized. As a drawback of this theoretically sound but stylized approach, behavior of our agents exhibits several unrealistic features to the effect that the present version of the model clearly lacks in realistic appeal. In particular, convergence of the Bayesian updating process of neo-additive capacities happens very

\footnotetext{
${ }^{11}$ Rabin (1998) reports empirical studies which do not support the conjecture that learning necessarily decreases biases. The models of biased Bayesian learning developed in Zimper and Ludwig (2009) and Zimper $(2009,2011)$ describe an agent's posterior neo-additive estimator for the likelihood of an event as the conditional Choquet expected value of the (unique) likelihood random variable taking on values in $[0,1]$. For alternative learning models with Bayesian overtones that may result in biased (i.e., ambiguous) longterm estimators see Epstein and Schneider $(2007,2008)$ who introduce multiple likelihoods and Epstein et al. (2010) who consider behaviorally motivated ad hoc deviations from rational Bayesian learning.
} 
quickly so that the agents of our model soon go for the extrema of a maximal and a minimal asset value whenever they have observed a sufficiently long sequence of good, respectively bad, news. Consequently, price patterns generated by our model crucially depend on the specification of such maximal and minimal asset values whereby such specification might amount to a rather arbitrary exercise. ${ }^{12}$ We also think that real-life agents - even behaviorally biased ones - are far more moderate than it is implied by our model and do not switch between such possible extrema just because some sequence of news triggers an optimistic or pessimistic reaction. We therefore regard it as an important avenue for future research to develop a sound decision-theoretic framework that has more behavioral appeal than the neo-additive framework. The main challenge will consist in keeping such a more realistic framework as parsimonious as possible.

\section{Concluding remarks and outlook}

Based on decision-theoretic first principles we introduce a behavioral model of asset price fluctuations which violates the rational beliefs paradigm in two different ways. First, agents have an incorrect world view because - due to their confirmatory bias - their subjective beliefs about economic fundamentals do not coincide with the objective distribution of these fundamentals regardless of how much information the agents receive. Second, agents incorrectly assume that their future selves interpret new information in the same way as they do. As an interesting feature, our model generates equilibrium price patterns that reflect underreaction of asset prices with respect to one period of good news as well as overreaction with respect to several periods of good news. These empirical phenomena have received substantial attention in the behavioral finance literature. In contrast to our approach, however, previous formal explanations for these phenomena were rather ad hoc and have not been derived from decision-theoretic first principles.

The key message of this paper is to illustrate how such pricing phenomena emerge from a non-standard model of Bayesian updating with a sound decision theoretic basis. Different Bayesian update rules for non-additive beliefs give rise to different pricing regimes: an optimistic, a pessimistic, and an "in between" full Bayesian pricing regime. Furthermore, our

\footnotetext{
${ }^{12}$ In a different application, Ludwig and Zimper (2012) relax such a convergence result by postulating limited memory. Using an equivalent assumption here would imply that prices in the optimistic, respectively pessimistic, regime would be determined by some weighted average between the maximal, respectively minimal, asset value and the rational expectations outcome. Qualitatively our results would, however, not change.
} 
confirmatory bias heuristic governs switches between these regimes. Our formal approach gives rise to model-endogenous concepts of "optimistic overpricing " and "pessimistic underpricing". The benchmark case of "correct" prices obtains whenever the agents' non-additive beliefs effectively reduce to an additive probability measure. As a consequence, the EMH is satisfied in our model if and only if the agents' subjective beliefs do not express any ambiguity with respect to the true stochastic process driving economic fundamentals.

Our current work extends this framework to develop a joint model for asset pricing anomalies such as the equity premium and excess volatility puzzles (Mehra and Prescott 1985; Shiller 1981). As such, our current research focuses on a joint theory for normal times, i.e., periods with standard asset price fluctuations, and exceptional times, i.e., periods with sizable booms and busts. 


\section{A Appendix}

\section{A.1 Deriving the equilibrium price process}

Observe that -independent of the specific choice of $\left(z_{t}, z_{t+1}, \ldots\right)$ - the maximal, respectively minimal, economic outcome occurs for every portfolio-choice in the same state $\left(\max Y_{1}, \max Y_{2}, \ldots\right) \in$ $\Omega$ resp. $\left(\min Y_{1}, \min Y_{2}, \ldots\right) \in \Omega$. Because of this fact and because of our assumption that every agent $I_{t}$ assumes that $\nu^{I_{t}}(\cdot \mid \cdot)$ governs the beliefs of his future selves, we can transform the neo-additive Choquet expected utility maximization problem into an equivalent (standard) expected utility maximization problem for conveniently constructed additive probability measures $\pi_{\text {opt }}^{I_{t}}$, $\pi_{\text {pess }}^{I_{t}}$ and $\pi_{F B}^{I_{t}}$. We thereby obtain the following equivalent maximization problems for the respective pricing regimes.

Optimistic pricing regime:

$$
\begin{aligned}
& \max _{\left(z_{t}, z_{t+1}, \ldots\right)} u\left(c_{t}\right)+E\left[\sum_{s=t+1}^{\infty} \beta^{s-t} u\left(c_{s}\right), \nu^{I_{t}}(\cdot \mid \cdot)\right] \\
= & \max _{\left(z_{t}, z_{t+1}, \ldots\right)} u\left(c_{t}\right)+E\left[\sum_{s=t+1}^{\infty} \beta^{s-t} u\left(c_{s}\right), \pi_{\text {opt }}^{I_{t}}\left(y_{s+1} \mid y_{0}, . ., y_{s}\right)\right]
\end{aligned}
$$

such that

$$
\pi_{o p t}^{I_{t}}\left(y_{s+1} \mid y_{0}, . ., y_{s}\right)= \begin{cases}\delta_{o p t}^{I_{t}}+\left(1-\delta_{o p t}^{I_{t}}\right) \cdot \pi\left(y_{s+1} \mid y_{0}, . ., y_{s}\right) & \text { for } y_{s+1}=\max Y_{s+1} \\ \left(1-\delta_{o p t}^{I_{t}}\right) \cdot \pi\left(y_{s+1} \mid y_{0}, . ., y_{s}\right) & \text { for } y_{s+1}<\max Y_{s+1}\end{cases}
$$

Pessimistic pricing regime:

$$
\begin{aligned}
& \max _{\left(z_{t}, z_{t+1}, \ldots\right)} u\left(c_{t}\right)+E\left[\sum_{s=t+1}^{\infty} \beta^{s-t} u\left(c_{s}\right), \nu^{I_{t}}(\cdot \mid \cdot)\right] \\
= & \max _{\left(z_{t}, z_{t+1}, \ldots\right)} u\left(c_{t}\right)+E\left[\sum_{s=t+1}^{\infty} \beta^{s-t} u\left(c_{s}\right), \pi_{\text {pess }}^{I_{t}}\left(y_{s+1} \mid y_{0}, . ., y_{s}\right)\right]
\end{aligned}
$$

such that

$$
\pi_{\text {pess }}^{I_{t}}\left(y_{s+1} \mid y_{0}, . ., y_{s}\right)= \begin{cases}\delta_{\text {pess }}^{I_{t}}+\left(1-\delta_{\text {pess }}^{I_{t}}\right) \cdot \pi\left(y_{s+1} \mid y_{0}, . ., y_{s}\right) & \text { if } y_{s+1}=\min Y_{s+1} \\ \left(1-\delta_{\text {pess }}^{I_{t}}\right) \cdot \pi\left(y_{s+1} \mid y_{0}, . ., y_{s}\right) & \text { if } y_{s+1}>\min Y_{s+1}\end{cases}
$$

Full Bayesian pricing regime:

$$
\begin{aligned}
& \max _{\left(z_{t}, z_{t+1}, \ldots\right)} u\left(c_{t}\right)+E\left[\sum_{s=t+1}^{\infty} \beta^{s-t} u\left(c_{s}\right), \nu^{I_{t}}(\cdot \mid \cdot)\right] \\
= & \max _{\left(z_{t}, z_{t+1}, \ldots\right)} u\left(c_{t}\right)+E\left[\sum_{s=t+1}^{\infty} \beta^{s-t} u\left(c_{s}\right), \pi_{F B}^{I_{t}}\left(y_{s+1} \mid y_{0}, . ., y_{s}\right)\right]
\end{aligned}
$$


such that

$$
\begin{aligned}
& \pi_{F B}^{I_{t}}\left(y_{s+1} \mid y_{0}, . ., y_{s}\right) \\
& \begin{cases}\delta_{F B}^{I_{t}} \cdot \lambda+\left(1-\delta_{F B}^{I_{t}}\right) \cdot \pi\left(y_{s+1} \mid y_{0}, . ., y_{s}\right) & \text { if } y_{s+1}=\max Y_{s+1} \\
\left(1-\delta_{F B}^{I_{t}}\right) \cdot \pi\left(y_{s+1} \mid y_{0}, . ., y_{s}\right) & \text { if } \min Y_{s+1}<y_{s+1}<\max Y_{s+1} \\
\delta_{F B}^{I_{t}} \cdot(1-\lambda)+\left(1-\delta_{F B}^{I_{t}}\right) \cdot \pi\left(y_{s+1} \mid y_{0}, . ., y_{s}\right) & \text { if } y_{s+1}=\min Y_{s+1} .\end{cases}
\end{aligned}
$$

Given this transformation of the CEU into an EU maximization problem the above results follow readily from standard arguments. For sake of completeness we demonstrate this for the optimistically biased pricing regime. Consider an optimistically biased agent so that

$$
\begin{aligned}
& E\left[\sum_{s=t+1}^{\infty} \beta^{s-t} u\left(c_{s}\right), \nu^{I_{t}}(\cdot \mid \cdot)\right] \\
= & \delta_{\text {opt }}^{I_{t}} \cdot \max _{\omega \in I_{t}} \sum_{s=t+1}^{\infty} \beta^{s-t} u\left(c_{s}\right)+\left(1-\delta_{\text {opt }}^{I_{t}}\right) \cdot E\left[\sum_{s=t+1}^{\infty} \beta^{s-t} u\left(c_{s}\right), \pi\left(y_{s+1} \mid y_{0}, . ., y_{s}\right)\right] .
\end{aligned}
$$

The corresponding period $s$ first order conditions, evaluated at equilibrium allocation $z_{t}^{*}=1$ for all $t$, imply

$$
\begin{aligned}
p_{s}^{*}= & \delta_{o p t}^{I_{t}} \cdot \beta \cdot \frac{u^{\prime}\left(\max Y_{s+1}\right)}{u^{\prime}\left(y_{s}\right)} \cdot\left(\max Y_{s+1}+p_{s+1}^{*}\right) \\
& +\left(1-\delta_{o p t}^{I_{t}}\right) \cdot E\left[\beta \cdot \frac{u^{\prime}\left(Y_{s+1}\right)}{u^{\prime}\left(y_{s}\right)} \cdot\left(Y_{s+1}+p_{s+1}^{*}\right), \pi\left(y_{s+1} \mid y_{0}, . ., y_{s}\right)\right] \\
= & \delta_{o p t}^{I_{t}} \cdot \min M_{s, s+1} \cdot\left(\max Y_{s+1}+p_{s+1}^{*}\right) \\
& +\left(1-\delta_{o p t}^{I_{t}}\right) \cdot E\left[M_{s, s+1} \cdot\left(Y_{s+1}+p_{s+1}^{*}\right), \pi\left(y_{s+1} \mid y_{0}, . ., y_{s}\right)\right]
\end{aligned}
$$

for all $s \geq t$. Notice that

$$
\min M_{s, s+1}=\beta \cdot \frac{u^{\prime}\left(\max Y_{s+1}\right)}{u^{\prime}\left(y_{s}\right)}
$$

follows from concavity of $u$. As a consequence, any period $t$ equilibrium asset price $p_{t}^{o p t}$ is characterized by the following system of equations

$$
\begin{aligned}
p_{s}^{o p t} & =E\left[M_{s, s+1} \cdot\left(Y_{s+1}+p_{s+1}^{o p t}\right), \pi_{o p t}^{I_{t}}\left(y_{s+1} \mid y_{0}, . ., y_{s}\right)\right] \\
& \equiv E_{s}^{o p t}\left[M_{s, s+1} \cdot\left(Y_{s+1}+p_{s+1}^{o p t}\right)\right]
\end{aligned}
$$

for all $s \geq t$ with $\pi_{o p t}^{I_{t}}\left(y_{s+1} \mid y_{0}, . ., y_{s}\right)$ defined above. Substitute

$$
p_{t+1}^{o p t}=E_{t+1}^{o p t}\left[m_{t+1, t+2} \cdot\left(Y_{t+2}+p_{t+2}^{o p t}\right)\right]
$$


in

$$
p_{t}^{o p t}=E_{t}^{o p t}\left[M_{t, t+1} \cdot\left(Y_{t+1}+p_{t+1}^{o p t}\right)\right]
$$

and observe that

$$
\begin{aligned}
p_{t}^{o p t} & =E_{t}^{o p t}\left[M_{t, t+1} \cdot\left(Y_{t+1}+E_{t+1}^{o p t}\left[M_{t+1, t+2} \cdot\left(Y_{t+2}+p_{t+2}^{o p t}\right)\right]\right)\right] \\
& =E_{t}^{o p t}\left[M_{t, t+1} \cdot Y_{t+1}\right]+E_{t}^{o p t}\left[M_{t, t+1} \cdot E_{t+1}^{o p t}\left[M_{t+1, t+2} \cdot\left(Y_{t+2}+p_{t+2}^{o p t}\right)\right]\right] \\
& =E_{t}^{o p t}\left[M_{t, t+1} \cdot Y_{t+1}\right]+E_{t}^{o p t}\left[E_{t+1}^{o p t}\left[M_{t, t+1} \cdot M_{t+1, t+2} \cdot\left(Y_{t+2}+p_{t+2}^{o p t}\right)\right]\right] \\
& =E_{t}^{o p t}\left[M_{t, t+1} \cdot Y_{t+1}\right]+E_{t}^{o p t}\left[E_{t+1}^{o p t}\left[M_{t, t+2} \cdot\left(Y_{t+2}+p_{t+2}^{o p t}\right)\right]\right] \\
& =E_{t}^{o p t}\left[M_{t, t+1} \cdot Y_{t+1}\right]+E_{t}^{o p t}\left[M_{t, t+2} \cdot Y_{t+2}\right]+E_{t}^{o p t}\left[M_{t, t+2} \cdot p_{t+2}^{o p t}\right] .
\end{aligned}
$$

The third line results from the fact that the random variable $M_{t, t+1}$ is a constant with respect to any given $E_{t+1}^{o p t}$ and the fifth line is implied by the law of iterative expectations for additive probability measures. Applying the same reasoning to $p_{t+2}^{o p t}, p_{t+2}^{o p t}, \ldots$ gives the desired result whenever the transversality condition is satisfied.

Remark. The key to the formal derivation of the equilibrium prices in the above proof is the fact that our assumptions allow us to transform the Choquet expected utility optimization problems into equivalent expected utility optimization problems. Hence, standard arguments such as sufficient characterization of global optima by first order conditions as well as the law of iterated expectations go through. This formal equivalence would break down if we had considered a portfolio choice problem with several assets that do not have a comonotonic payoff-structure. Technically speaking, the corresponding portfolio optimization problem would then exhibit kinks so that first order conditions are no longer sufficient criteria for global optima.

\section{A.2 Formal proof of the proposition}

Step 1. Observe that

$$
\begin{aligned}
E\left[Y_{t+1}, \pi\left(\cdot \mid y_{t-j-1}>y_{t-j}<\ldots<y_{t}\right)\right] & =E\left[Y_{t+1}, \pi\left(\cdot \mid y_{t-j-1}<y_{t-1}>\ldots>y_{t}\right)\right] \\
& =E\left[Y_{t+1}, \pi\right]
\end{aligned}
$$

for $j \geq 1$, by the independence assumption. 
Step 2. Fix some $n$ and consider some $j<n$. Then $y_{t-j-1}>y_{t-j}<\ldots<y_{t}$ implies $p_{t}^{*}=p_{t}^{F B}$ so that

$$
\begin{aligned}
& E\left[\frac{p_{t+1}^{*}+Y_{t+1}}{p_{t}^{*}}, \pi\left(\cdot \mid y_{t-j-1}>y_{t-j}<\ldots<y_{t}\right)\right] \\
= & \frac{E\left[p_{t+1}^{*}, \pi\left(\cdot \mid y_{t-j-1}>y_{t-j}<\ldots<y_{t}\right)\right]+E\left[Y_{t+1}, \pi\right]}{p_{t}^{F B}} .
\end{aligned}
$$

Similarly, $y_{t-j-1}<y_{t-j}>\ldots>y_{t}$ implies

$$
\begin{aligned}
& E\left[\frac{p_{t+1}^{*}+Y_{t+1}}{p_{t}^{*}}, \pi\left(\cdot \mid y_{t-j-1}<y_{t-j}>\ldots>y_{t}\right)\right] \\
= & \frac{E\left[p_{t+1}^{*}, \pi\left(\cdot \mid y_{t-j-1}<y_{t-j}>\ldots>y_{t}\right)\right]+E\left[Y_{t+1}, \pi\right]}{p_{t}^{F B}} .
\end{aligned}
$$

Consequently,

$$
E\left[R_{t+1}, \pi\left(\cdot \mid y_{t-j-1}>y_{t-j}<\ldots<y_{t}\right)\right]>E\left[R_{t+1}, \pi\left(\cdot \mid y_{t-j-1}<y_{t-j}>\ldots>y_{t}\right)\right]
$$

is satisfied, if and only if,

$$
E\left[p_{t+1}^{*}, \pi\left(\cdot \mid y_{t-j-1}>y_{t-j}<\ldots<y_{t}\right)\right]>E\left[p_{t+1}^{*}, \pi\left(\cdot \mid y_{t-j-1}<y_{t-j}>\ldots>y_{t}\right)\right] .
$$

Step 3. Consider the case $j<n-1$. Then $p_{t+1}^{*}=p_{t+1}^{F B}$ for good as well as for bad news so that

$$
\begin{aligned}
E\left[p_{t+1}^{*}, \pi\left(\cdot \mid y_{t-j-1}>y_{t-j}<\ldots<y_{t}\right)\right] & =E\left[p_{t+1}^{*}, \pi\left(\cdot \mid y_{t-j-1}<y_{t-j}>\ldots>y_{t}\right)\right] \\
& \Leftrightarrow \\
E\left[R_{t+1}, \pi\left(\cdot \mid y_{t-j-1}>y_{t-j}<\ldots<y_{t}\right)\right] & =E\left[R_{t+1}, \pi\left(\cdot \mid y_{t-j-1}<y_{t-j}>\ldots>y_{t}\right)\right] .
\end{aligned}
$$

Consequently, there is neither underreaction nor overreaction. This proves (26).

Step 4. Consider the case $j=n-1$. Then $p_{t+1}^{*}$ results for good news in period $t$ either from the optimistic or from the full Bayesian pricing regime, i.e.,

$$
\begin{aligned}
& E\left[p_{t+1}^{*}, \pi\left(\cdot \mid y_{t-j-1}>y_{t-j}<\ldots<y_{t}\right)\right] \\
= & E\left[p_{t+1}^{o p t}, \pi\left(\cdot \mid y_{t-j-1}>y_{t-j}<\ldots<y_{t}<y_{t+1}\right)\right] \cdot \pi\left(y_{t}<y_{t+1}\right) \\
& +E\left[p_{t+1}^{F B}, \pi\left(\cdot \mid y_{t-j-1}>y_{t-j}<\ldots<y_{t} \geq y_{t+1}\right)\right] \cdot \pi\left(y_{t} \geq y_{t+1}\right)
\end{aligned}
$$

For bad news in period $t, p_{t+1}^{*}$ follows either from the pessimistic or from the full Bayesian pricing regime, i.e.,

$$
\begin{aligned}
& E\left[p_{t+1}^{*}, \pi\left(\cdot \mid y_{t-j-1}<y_{t-j}>\ldots>y_{t}\right)\right] \\
= & E\left[p_{t+1}^{\text {pess }}, \pi\left(\cdot \mid y_{t-j-1}<y_{t-j}>\ldots>y_{t}>y_{t+1}\right)\right] \cdot \pi\left(y_{t}>y_{t+1}\right) \\
& +E\left[p_{t+1}^{F B}, \pi\left(\cdot \mid y_{t-j-1}<y_{t-j}>\ldots>y_{t} \leq y_{t+1}\right)\right] \cdot \pi\left(y_{t} \leq y_{t+1}\right) .
\end{aligned}
$$


If the histories satisfy condition (27), then

$$
\begin{aligned}
\pi\left(y_{t-j-1}>y_{t-j}<\ldots<y_{t}<y_{t+1 t}\right) & >0 \text { or } \\
\pi\left(y_{t-j-1}<y_{t-j}>\ldots>y_{t}>y_{t+1}\right) & >0 .
\end{aligned}
$$

Together with (23), the lhs in (34) first-order stochastically dominates the lhs in (34) so that we obtain underreaction for $j=n-1$. If, instead, (28) holds, then

$$
\begin{aligned}
& \pi\left(y_{t-j-1}>y_{t-j}<\ldots<y_{t}<y_{t+1}\right)=0 \text { and } \\
& \pi\left(y_{t-j-1}<y_{t-j}>\ldots>y_{t}>y_{t+1}\right)=0 .
\end{aligned}
$$

This proves (29).

Step 5. Consider the case $j \geq n$. Then $p_{t}^{*}=p_{t}^{o p t}$ for history $y_{t-j-1}>y_{t-j}<\ldots<y_{t}$ and $p_{t}^{*}=p_{t}^{p e s s}$ for history $y_{t-j-1}<y_{t-j}>\ldots>y_{t}$. Consequently,

$$
\begin{aligned}
& E\left[R_{t+1}, \pi\left(\cdot \mid y_{t-j-1}>y_{t-j}<\ldots<y_{t}\right)\right] \\
= & \frac{E\left[p_{t+1}^{*}, \pi\left(\cdot \mid y_{t-j-1}>y_{t-j}<\ldots<y_{t}\right)\right]+E\left[Y_{t+1}, \pi\right]}{p_{t}^{o p t}}
\end{aligned}
$$

and

$$
\begin{aligned}
& E\left[R_{t+1}, \pi\left(\cdot \mid y_{t-j-1}<y_{t-j}>\ldots>y_{t}\right)\right] \\
= & \frac{E\left[p_{t+1}^{*}, \pi\left(\cdot \mid y_{t-j-1}<y_{t-j}>\ldots>y_{t}\right)\right]+E\left[Y_{t+1}, \pi\right]}{p_{t}^{\text {pess }}} .
\end{aligned}
$$

Because

$$
\frac{E\left[Y_{t+1}, \pi\right]}{p_{t}^{o p t}}<\frac{E\left[Y_{t+1}, \pi\right]}{p_{t}^{\text {pess }}}
$$

our model generates overreaction (3) if the sufficient condition

$$
\frac{E\left[p_{t+1}^{*}, \pi\left(\cdot \mid y_{t-j-1}>y_{t-j}<\ldots<y_{t}\right)\right]}{p_{t}^{\text {opt }}} \leq \frac{E\left[p_{t+1}^{*}, \pi\left(\cdot \mid y_{t-j-1}<y_{t-j}>\ldots>y_{t}\right)\right]}{p_{t}^{\text {pess }}}
$$

is satisfied. If $j \geq n, p_{t+1}^{*}$ results for good news in period $t$ either from the optimistic or full Bayesian pricing regime, i.e.,

$$
\begin{aligned}
& E\left[p_{t+1}^{*}, \pi\left(\cdot \mid y_{t-j-1}>y_{t-j}<\ldots<y_{t}\right)\right] \\
= & E\left[p_{t+1}^{o p t}, \pi\left(\cdot \mid y_{t-j-1}>y_{t-j}<\ldots<y_{t}<y_{t+1}\right)\right] \cdot \pi\left(y_{t}<y_{t+1}\right) \\
& +E\left[p_{t+1}^{F B}, \pi\left(\cdot \mid y_{t-j-1}>y_{t-j}<\ldots<y_{t} \geq y_{t+1}\right)\right] \cdot \pi\left(y_{t} \geq y_{t+1}\right) .
\end{aligned}
$$


Accordingly, $p_{t+j}^{*}$ results for bad news either from the pessimistic or full Bayesian pricing regime, i.e.,

$$
\begin{aligned}
& E\left[p_{t+1}^{*}, \pi\left(\cdot \mid y_{t-j-1}<y_{t-j}>\ldots>y_{t}\right)\right] \\
= & E\left[p_{t+1}^{\text {pess }}, \pi\left(\cdot \mid y_{t-j-1}<y_{t-j}>\ldots>y_{t}>y_{t+1}\right)\right] \cdot \pi\left(y_{t}>y_{t+1}\right) \\
& +E\left[p_{t+1}^{F B}, \pi\left(\cdot \mid y_{t-j-1}<y_{t-j}>\ldots>y_{t} \leq y_{t+1}\right)\right] \cdot \pi\left(y_{t} \leq y_{t+1}\right) .
\end{aligned}
$$

Substituting in inequality (35) and rearranging gives

$$
\begin{aligned}
& \left(\frac{E\left[p_{t+1}^{\text {opt }}, \pi\left(\cdot \mid y_{t-j-1}>y_{t-j}<\ldots<y_{t}<y_{t+1}\right)\right]}{p_{t}^{\text {opt }}}-\frac{E\left[p_{t+1}^{F B}, \pi\left(\cdot \mid y_{t-j-1}<y_{t-j}>\ldots>y_{t} \leq y_{t+1}\right)\right]}{p_{t}^{\text {pess }}}\right. \\
& \cdot \pi\left(y_{t}<y_{t+1}\right) \\
\leq & \left(\frac{E\left[p_{t+1}^{\text {pess }}, \pi\left(\cdot \mid y_{t-j-1}<y_{t-j}>\ldots>y_{t}>y_{t+1}\right)\right]}{p_{t}^{\text {pess }}}-\frac{E\left[p_{t+1}^{F B}, \pi\left(\cdot \mid y_{t-j-1}>y_{t-j}<\ldots<y_{t}>y_{t+1}\right)\right]}{p_{t}^{\text {opt }}}\right. \\
& \cdot \pi\left(y_{t}>y_{t+1}\right) \\
& +\left(\frac{E\left[p_{t+1}^{F B}, \pi\left(\cdot \mid y_{t-j-1}<y_{t-j}>\ldots>y_{t}=y_{t+1}\right)\right]}{p_{t}^{\text {pess }}}-\frac{E\left[p_{t+j}^{F B}, \pi\left(\cdot \mid y_{t-j-1}>y_{t-j}<\ldots<y_{t}=y_{t+j}\right)\right.}{p_{t}^{\text {opt }}}\right. \\
& \cdot \pi\left(y_{t}=y_{t+1}\right)
\end{aligned}
$$

By the i.i.d. assumption, $\lim _{t \rightarrow \infty} \pi\left(I_{t}\right)=0$ for all $I_{t}$, which implies

$$
\lim _{t \rightarrow \infty} \delta_{\text {opt }}^{I_{t}}=\lim _{t \rightarrow \infty} \delta_{\text {pess }}^{I_{t}}=\lim _{t \rightarrow \infty} \delta_{F B}^{I_{t}}=1 .
$$

As a consequence, the respective equilibrium prices of any given regime converge to some constant, i.e.,

$$
\begin{aligned}
\lim _{t \rightarrow \infty} p_{t}^{o p t} & =\frac{\beta}{1-\beta} \cdot \max Y, \\
\lim _{t \rightarrow \infty} p_{t}^{\text {pess }} & =\frac{\beta}{1-\beta} \cdot \min Y, \\
\lim _{t \rightarrow \infty} p_{t}^{F B} & =\frac{\beta}{1-\beta} \cdot(\lambda \cdot \max Y+(1-\lambda) \cdot \min Y) .
\end{aligned}
$$

By continuity, the expected prices in (36) satisfy

$$
\begin{aligned}
E\left[p_{t+1}^{o p t}, \pi(\cdot \mid \cdot)\right] & \simeq p_{t}^{o p t} \simeq \frac{\beta}{1-\beta} \cdot \max Y, \\
E\left[p_{t+1}^{F B}, \pi(\cdot \mid \cdot)\right] & \simeq p_{t}^{F B} \simeq \frac{\beta}{1-\beta} \cdot(\lambda \cdot \max Y+(1-\lambda) \cdot \min Y) \\
E\left[p_{t+1}^{\text {pess }}, \pi(\cdot \mid \cdot)\right] & \simeq p_{t}^{\text {pess }} \simeq \frac{\beta}{1-\beta} \cdot \min Y,
\end{aligned}
$$


for sufficiently large $t$. Substituting in (36) gives, for sufficiently large $t$,

$$
\begin{aligned}
& \left(1-\frac{p_{t}^{F B}}{p_{t}^{\text {pess }}}\right) \cdot \pi\left(y_{t}<y_{t+1}\right) \leq\left(1-\frac{p_{t}^{F B}}{p_{t}^{\text {opt }}}\right) \cdot \pi\left(y_{t}>y_{t+1}\right) \\
& +\left(\frac{p_{t}^{F B}}{p_{t}^{\text {pess }}}-\frac{p_{t}^{F B}}{p_{t}^{\text {opt }}}\right) \cdot \pi\left(y_{t}=y_{t+1}\right)
\end{aligned}
$$

which is always satisfied with strict inequality since the lhs is negative and the rhs is positive because of $p_{t}^{\text {opt }}>p_{t}^{F B}>p_{t}^{\text {pess }}$. This proves overreaction for $j \geq n$ if $t$ is sufficiently large. $\square$ 


\section{References}

Antweiler, W,. and M.Z. Frank (2006) "Do U.S. Stock Markets Typically Overreact to Corporate News Stories?," Working Paper.

Anscombe, F.J., and R.J. Aumann (1963) "A Definition of Subjective Probability," Annals of American Statistics 34, 199-205.

Barberis, N., Shleifer, A., and R. Vishny (1998) "A Model of Investor Sentiment," Journal of Financial Economics 49, 307-343.

Benartzi, S., and R.H. Thaler (1995) "Myopic Loss Aversion and the Equity Premium Puzzle," Quarterly Journal of Economics 110, 73-92.

Bernard, V. (1992) "Stock Price Reactions to Earnings Announcements," in: Thaler, R. (Ed.), Advances in Behavioral Finance pp. 303-340, Russell Sage Foundation: New York.

Bernard, V., and J. Thomas (1990) "Evidence that Stock Prices Do Not Fully Reflect the Implications of Current Earnings for Future Earnings," Journal of Accounting and Economics 13, 305-341.

Burks, A.W. (1977) Chance, Cause, Reason: An Inquiry into the Nature of Scientific Evidence The University of Chicago Press, Chicago

Chan, W.S. (2003) "Stock Price Reaction to News and No-News: Drift and Reversal after Headline," Journal of Financial Economics 70, 223-260.

Chan, L., Jegadeesh, N., and J. Lakonishok (1997) "Momentum Strategies," Journal of Finance 51, 1681-1713.

Chateauneuf, A., Eichberger, J., and S. Grant (2007) "Choice under Uncertainty with the Best and Worst in Mind: Neo-additive Capacities," Journal of Economic Theory 127, 538-567.

Chopra, N., Lakonishok, J., and J. Ritter (1992) "Measuring Abnormal Performance: Do Stocks Overreact?," Journal of Financial Economics 31, 235-268.

Cohen, M., Gilboa, I., Jaffray J.Y., and D. Schmeidler (2000) "An Experimental Study of Updating Ambiguous Beliefs," Risk, Decision, and Policy 5, 123-133. 
Cutler, D., Poterba, J., and L. Summers (1991) "Speculative Dynamics," Review of Economic Studies 58, 529-546.

Daniel, K., Hirshleifer, D., and A. Subrahmanyam (1998) "Investor Psychology and Security Market Under- and Overreactions," Journal of Finance 53, 1839-1885.

De Bondt, W., and R. Thaler (1985) "Does the Stock Market Overreact?," Journal of Finance 40, 793-808.

Eichberger, J., and D. Kelsey (1999) "E-Capacities and the Ellsberg Paradox," Theory and Decision 46, 107-140.

Eichberger, J., Grant, S., and D. Kelsey (2006) "Updating Choquet Beliefs," Journal of Mathematical Economics 43, 888-899.

Ellsberg, D. (1961) "Risk, Ambiguity and the Savage Axioms," Quarterly Journal of Economics 75, 643-669.

Epstein, L.G. (1999) "A Definition of Uncertainty Aversion," The Review of Economic Studies 66, 579-608.

Epstein, L.G. and M. Schneider (2003) "Recursive Multiple-Priors," Journal of Economic Theory 113, 1-31.

Epstein, L. G., and M. Schneider (2007) "Learning under Ambiguity," Review of Economic Studies 74, 1275-1303.

Epstein, L. G., and M. Schneider (2008) "Ambiguity, Information Quality, and Asset Pricing," The Journal of Finance 63, 197-228.

Epstein, L.G. and T. Wang (1994) "Intertemporal Asset Pricing Under Knightian Uncertainty," Econometrica 62, 283-322.

Epstein, L.G., Noor, J., and A. Sandroni (2010) "Non-Bayesian Learning," The B.E. Journal of Theoretical Economics (Advances), 10 (1).

Ghirardato, P. (2002) "Revisiting Savage in a Conditional World," Economic Theory 20, 83-92.

Ghirardato, P., and M. Marinacci (2002) "Ambiguity Made Precise: A Comparative Foundation," Journal of Economic Theory 102, 251-289. 
Gilboa, I. (1987) "Expected Utility with Purely Subjective Non-Additive Probabilities," Journal of Mathematical Economics 16, 65-88.

Gilboa, I. and D. Schmeidler (1989) "Maxmin Expected Utility with Non-Unique Priors" Journal of Mathematical Economics 18, 141-153.

Gilboa, I., and D. Schmeidler (1993) "Updating Ambiguous Beliefs," Journal of Economic Theory 59, 33-49.

Hammond, P.J. (1988) "Consequentialist Foundation for Expected Utility," Theory and Decision 25, 25-78.

Hansen, L.P., Sargent, T.J., Turmuhambetova, G. and N. Williams (2006) "Robust Control and Model Misspecification" Journal of Economic Theory 128, 45-90.

Hong, H., and J.C. Stein (1999) "A Unified Theory of Underreaction, Momentum Trading, and Overreaction in Asset Markets," Journal of Finance 54, 2143-2184.

Jegadeesh, N., and S. Titman (1993) "Returns to Buying Winners and Selling Losers: Implications for Stock Market Efficiency," Journal of Finance 48, 65-91.

Kahneman, D., and A. Tversky (1979) "Prospect Theory: An Analysis of Decision under Risk," Econometrica 47, 263-291.

Karni, E., and Z. Safra (1990) "Behaviorally Consistent Optimal Stopping Rules," Journal of Economic Theory 51, 391-402.

Kreps, D.M., and R. Wilson (1982) "Sequential Equilibria," Econometrica 50, 863-894.

La Porta, R. (1996) "Expectations and the Cross-section of Returns," Journal of Finance 51, 1715-1742.

La Porta, R., Lakonishok, J., Shleifer, A., and R. Vishny (1997) "Good News for Value Stocks: Further Evidence on Market Efficiency," Journal of Finance 52, 859-874.

LeBaron, B. (2000) "Agent-based Computational Finance: Suggested Readings and Early Research," Journal of Economic Dynamics and Control 24, 679-702.

LeBaron, B. (2006) "Agent-based Computational Finance," In: Tesfatsion, L., and K.L. Judd (ed.), Handbook of Computational Economics 2 (Ch. 4). 
Lucas, R.E.Jr. (1978) "Asset Prices in an Exchange Economy," Econometrica 46, 14291445 .

Ludwig, A. and A. Zimper (2011) "A Parsimonious Model of Subjective Life-Expectancy, "Working Paper.

Ludwig, A. and A. Zimper (2012) "Biased Bayesian Learning with an Application to the Risk-free Rate Puzzle, " Working Paper.

Machina, M. (1989) "Dynamic Consistency and Non-Expected Utility Models of Choice Under Uncertainty," Journal of Economic Literature 27, 1622-1668.

Mehra, R., and E.C. Prescott (1985) "The Equity Premium: A Puzzle," Journal of Monetary Economics 15, 145-161.

Pires, C.P. (2002) "A Rule for Updating Ambiguous Beliefs," Theory and Decision 53, $137-152$.

Pollak, R.A. (1968) "Consistent Planning," The Review of Economic Studies 35, 201-208.

Quiggin, J.P. (1981) "Risk Perception and Risk Aversion among Australian Farmers," Australian Journal of Agricultural Economics 25, 160-169.

Quiggin, J.P. (1982) "A Theory of Anticipated Utility," Journal of Economic Behavior and Organization 3, 323-343.

Rabin, M. (1998) "Psychology and Economics," Journal of Economic Literature 36, 11-46.

Rouwenhorst, G. (1997) "International Momentum Strategies," Journal of Finance 53, $267-284$.

Sarin, R., and P.P. Wakker (1998a) "Revealed Likelihood and Knightian Uncertainty" Journal of Risk and Uncertainty 16, 223-250.

Sarin, R., and P.P. Wakker (1998b) "Dynamic Choice and NonExpected Utility" Journal of Risk and Uncertainty 17, 87-119.

Savage, L.J. (1954) The Foundations of Statistics, John Wiley and \& Sons, Inc.: New York, London, Sydney. 
Schmeidler, D. (1986) "Integral Representation without Additivity," Proceedings of the American Mathematical Society 97, 255-261.

Schmeidler, D. (1989) "Subjective Probability and Expected Utility without Additivity," Econometrica 57, 571-587.

Shiller, R.J. (1981) "Do Stock Prices Move too Much to be Justified by Subsequent Changes in Dividends?," American Economic Review 71, 421-498.

Siniscalchi, M. (2011) "Dynamic Choice under Ambiguity," Theoretical Economics 6, 379421.

Strotz, R.H. (1956) "Myopia and Inconsistency in Dynamic Utility Maximization," The Review of Economic Studies 23, 165-180.

Thaler, R. H., Tversky, A., Kahneman, D., and A. Schwartz (1997) "The Effect of Myopia and Loss Aversion on Risk Taking: An Experimental Test," Quarterly Journal of Economics 112, 647-661.

Tversky, A., and D. Kahneman (1992) "Advances in Prospect Theory: Cumulative Representations of Uncertainty," Journal of Risk and Uncertainty 5, 297-323.

Wakker, P.P. (2001) "Testing and Characterizing Properties of Nonadditive Measures through Violations of the Sure-Thing Principle," Econometrica 69, 1039-1059.

Wakker, P.P. (2004) "On the Composition of Risk Preference and Belief," Psychological Review 111, 236-241.

Wakker, P.P. (2010) Prospect Theory: For Risk and Ambiguity Cambridge University Press: Cambridge, UK.

Wakker, P.P., and A. Tversky (1993) "An Axiomatization of Cumulative Prospect Theory," Journal of Risk and Uncertainty 7, 147-176.

Wu, G., and R. Gonzalez (1996) "Curvature of the Probability Weighting Function," Management Science 42, 1676-1690.

Wu, G., and R. Gonzalez (1999) "Nonlinear Decison Weighst in Choice under Uncertainty," Management Science 45, 74-85. 
Zarowin, P. (1989) "Does the Stock Market Overreact to Corporate Earnings Information?," Journal of Finance 44, 1385-1400.

Zimper, A. (2009) "Half Empty, Half Full and Why We Can Agree to Disagree Forever," Journal of Economic Behavior and Organization 71, 283-299.

Zimper, A. (2011) "Do Bayesians Learn their Way out of Ambiguity?," Decision Analysis 8, 269-285.

Zimper, A. (2012) "Asset Pricing in a Lucas Fruit-tree Economy with the Best and Worst in Mind," Journal of Economic Dynamics and Control 36, 610-628.

Zimper, A., and A. Ludwig (2009) "On Attitude Polarization Under Bayesian Learning With Non-additive Beliefs," Journal of Risk and Uncertainty 39, 181-212. 